\title{
Determination of the force systems produced by different configurations of tear drop orthodontic loops
}

\author{
Guilherme Thiesen¹, Roberto Hideo Shimizu², Caio Vinicius Martins do Valle? \\ Karyna Martins do Valle-Corotti ${ }^{4}$, Jefferson Ricardo Pereira ${ }^{5}$, Paulo Cesar Rodrigues Conti ${ }^{6}$
}

\begin{abstract}
Objective: To determine the mechanical characteristics of teardrop loop with and without helix fabricated using different metal alloy compositions (stainless steel and beta-titanium), submitted to different intensities of bends preactivation $\left(0^{\circ}\right.$ and $\left.40^{\circ}\right)$, and with different cross-sectional dimension of the wire used to build these loops $(0.017 \times 0.025$-in and 0.019 x 0.025-in). Methods: Eighty loops used to close spaces were submitted to mechanical tests. The magnitudes of horizontal force, the moment/force ratio, and the load/deflection ratio produced by the specimens were quantified. Loops were submitted to a total activation of $5.0 \mathrm{~mm}$ and the values were registered for each $1.0 \mathrm{~mm}$ of activation. For statistic data analysis, a analysis of variance was performed and a Tukey's Multiple Comparison test was used as supplement, considering a 5\% level of significance. Results: In general, teardrop loops with helix produced lower magnitudes of horizontal force and load/deflection ratio, and higher moment/force ratio than teardrop loops without helix. Among all analyzed variables, metal alloy composition presented greater influence in the horizontal force and in the load/deflection ratio. The moment/force ratio showed to be more influenced by the preactivation of loops for space closure.
\end{abstract}

Keywords: Biomechanics. Orthodontic space closure. Tooth extraction. Orthodontics.

Objetivo: determinar as características mecânicas de alças em gota e em gota com helicoide, quando da incorporação de variações na liga metálica (aço inoxidável e beta-titânio), na intensidade de dobras de pré-ativação $\left(0^{\circ}\right.$ e $\left.40^{\circ}\right)$ e na secção transversal do fio utilizado para a construção dessas alças $(0,017$ ” x 0,025 ” e 0,019 ” x 0,025 ”). Métodos: foram submetidas ao ensaio mecânico 80 alças para fechamento de espaços, sendo quantificadas as magnitudes de força horizontal, proporção momento/força e relação carga/deflexão produzidas pelos corpos de prova. As alças foram submetidas a uma ativação total de $5,0 \mathrm{~mm}$, sendo registrados os valores a cada $1,0 \mathrm{~mm}$ de ativação. No tratamento estatístico dos dados obtidos, foi realizada a análise de variância, sendo essa complementada pelo teste de comparações múltiplas de Tukey, considerando o nível de significância de 5\%. Resultados: de maneira geral, as alças em gota com helicoide produziram menores magnitudes de força horizontal e relação carga/deflexão e maiores valores de proporção momento/força do que as alças em gota. Dentre todas as variáveis analisadas, aquela que apresentou uma maior influência na força horizontal e na relação carga/deflexão produzidas pelas alças foi a liga metálica. Já a proporção momento/força mostrou ser influenciada em maior grau pela pré-ativação das alças de fechamento de espaços.

Palavras-chave: Biomecânica. Fechamento de espaço ortodôntico. Extração dentária. Ortodontia.

${ }^{1} \mathrm{MSc}$ in Orthodontics and Facial Orthopedic, PUCRS. Professor, Graduation and Post-graduation course in Orthodontics, UNISUL-SC and UNIASSELVI. ${ }^{2} \mathrm{MSc}$ and $\mathrm{PhD}$ in Orthodontics, UNESP-Araraquara. Professor of Orthodontics, Tuiuti University.

${ }^{3} \mathrm{MSc}$ in Orthodontics, FOB-USP. Coordinator of the specialization course in Orthodontics, Leonardo da Vinci University. PhD Student in Oral Rehabilitation, FOB-USP.

${ }^{4} \mathrm{MSc}$ and $\mathrm{PhD}$ in Orthodontics, FOB-USP. Associate Professor, Orthodontics, UNICID. Professor of the Master Program in Orthodontics, UNICID. ${ }^{5} \mathrm{MSc}$ and $\mathrm{PhD}$ in Oral Rehabilitation, FOB-USP. Professor, Graduation in Dentistry, UNISUL-SC.

${ }^{6}$ Head professor, Prosthesis, FOB-USP.
How to cite this article: Thiesen G, Shimizu RH, Valle CVM, Valle-Corotti KM, Pereira JR, Conti PCR. Determination of the force systems produced by different configurations of tear drop orthodontic loops. Dental Press J Orthod. 2013 MarApr;18(2):19.el-18

Submitted: April 20,2009 - Revised and accepted: June 06, 2009

Contact address: Guilherme Thiesen

Av. Madre Benvenuta, 1285 - Santa Mônica

CEP: 88.035 - 000 - Florianópolis / SC - Brazil

E-mail: guilhermethiesen@yahoo.com.br 


\section{INTRODUCTION}

Space closure in orthodontics, particularly those from tooth extractions, still represents a challenge to the professional in this area. It occurs mainly due to the increase in treatment time, patient discomfort, as well as the pursuit of excellence on the finishing stage. These factors are related to the method used to achieve such result.

During the development of orthodontics specialty, several devices have been elaborated to close these spaces. Such mechanisms present a wide variety of designs, thicknesses and wire configurations, source of force application, and also factors related to anchorage control. ${ }^{4}$ Among several devices described in the literature, there are a wide range of loops which once incorporated to continuous or segmented arches can be used for tooth movement. Current knowledge in biomechanics, allied to the development of new materials, made possible a great upgrading in space closure loops configuration, which has simplified the mechanics and improved tissue answers, minimizing the biological cost of treatment. , $3,6,21,24^{2}$

Due to the great number of mechanical options, lot of attention must be spent during the selection of more appropriate model for each case. Certain aspects must be analyzed in this choice, among them it can be quote the loop design, its quantity of activation, the kind of movement expected and the force system generated. The orthodontist must be aware that in most decisions, the application of a simple horizontal force on adjacent tooth to edentulous space does not provide an appropriate tooth positioning because the application of this kind of force implies in a lack of root parallelism at the end of the space closure. Then, it is necessary a concomitant application of a moment to oppose this rotational tendency. Therefore, it is very important that, when using the loops for space closure, the professional determine precisely the force system generated, in other words, it is necessary for the orthodontist to have the knowledge of horizontal force magnitude and the moment/force ratio exerted on the activation of these devices. ${ }^{16,22,28}$

Burstone $^{2}$ claimed there are three fundamental properties which characterize orthodontic space closure loops: 1) the moment/force $(\mathrm{M} / \mathrm{F})$ ratio which determine tooth rotation center and in this way allows root control during tooth movement; 2) horizontal force produced during the activation of this loop; and 3) load/deflection ratio, which define the loss in force magnitude following each millimeter of deactivation. The generation of this biomechanical system is performed more integrally, accurately, predictable, and easily through the use of pre-calibrated loops.

Among orthodontic devices used for space closure, teardrop loop and teardrop loop with helix, despite they are very studied and employed, 9,14,21,24,26,27,28 still present some limitations and questions as for clinical use. There are no papers in literature which describe the force system generated by these loops in different settings of the metal alloy used, of the cross-sectional dimension of orthodontic archwire, of the magnitude of preactivation, and of the activation itself. Most papers published on this topic did not evaluate the effects from different preactivation magnitudes, as well as the level of moment/force ratio generated during the activation of specimens or even the helix incorporation in its design.

The objective of the present study was to verify the effect of changes in teardrop configuration, such as the presence or absence of helix, the cross-section dimension of orthodontic archwire, incorporation of different preactivation in the loops, and also metal alloy composition used, aiming at investigating the interaction among these factors.

\section{PROPOSITION}

To analyze mechanical characteristics in different configuration of teardrop loops to space closure, submitting them to a $5.0-\mathrm{mm}$ activation, intending:

a) To verify if there are any difference between the force systems produced by teardrop loop with and without helix.

b) To verify the effect of some variables such as cross-sectional dimension of the orthodontic archwire, incorporation of preactivations, and metal alloy composition, in the force system produced by tested loops.

c) To determine among analyzed variables those which have more influence in the force systems produced.

\section{MATERIAL AND METHODS}

The sample consisted of 80 orthodontic loops for space closure made by the same operator. It was used 
stainless steel wire 18/8 from the commercial brand Unitek (3M Unitek Dental Products, Monrovia, CA - USA) and beta-titanium wires from the commercial brand TMA (Ormco Corporation, Glendora, CA - USA), with two different cross-section dimension, $0.017 \times 0.025$-in and $0.019 \times 0.025$-in.

Specimens were divided into two groups (Fig 1). For fixing the loops in the mechanical testing machine, their anterior (alpha) and posterior (beta) extremities presented additional extensions in their total length, according to the methodology previously described. ${ }^{25}$

The Group 1 (G1) comprised forty teardrop loops. These loops were divided regarding the metal alloy composition into two subgroups with twenty specimens each, then divided equally again into more two subgroups according to archwire cross-section dimension (Table 1). These space closure loops were built with $8 \mathrm{~mm}$ height and $4 \mathrm{~mm}$ in diameter, vertical rods touching each other in the base, and anterior (alpha) and posterior (beta) extremities with $10.5 \mathrm{~mm}$ each (Fig 1).

It was inserted two different total intensity of preactivation: $0^{\circ}$ and $40^{\circ}$. For a total preactivation of $40^{\circ}$, first it was inserted a preactivation of $20^{\circ}$ on the bend 2 and posteriorly activations of $10^{\circ}$ on the bends 1 and 3 (Fig 2), totalling $40^{\circ}$ of preactivation.

The Group 2 (G2) comprised forty teardrop loops made with an apical helix in their design. In this group, the loops were divided into two subgroups, where twenty specimens were used for each metal alloy, and divided again into more two subgroups of ten each, according to the cross-sectional dimension (Table 2). These space closure loops were built with $8 \mathrm{~mm}$ height and $4 \mathrm{~mm}$ in helix diameter, vertical rods touching each other in the base, both in anterior (alpha) and posterior (beta) extremities with $10.5 \mathrm{~mm}$ each (Fig 1). It was inserted two different total intensity of preactivation: $0^{\circ}$ and $40^{\circ}$. The preactivation bends were inserted in the same way of Group 1 (Fig 2).

As advised by Braun and Garcia, ${ }^{1}$ and Halazonetis, ${ }^{7}$ the preactivation bends were performed for all groups in a distributed way occlusogingivally, promoting an increase in the moment with no interference on the horizontal force magnitude. The preactivation bends were inserted in such way that the long vertical axis of each loop formed equal angles with their mesial and horizontal rods.

In order to standardize the shape and dimensions of the specimens in a same subgroup, as well as ensure that the preactivation bends would have the desired angles, it were made templates in graph paper (Fig 3), as well as templates in vinyl polysiloxane impression material of heavy body viscosity (Fig 4) from commercial brand Express (3M Dental Products, St. Paul, MN - USA).

In order to perform this mechanical test, it was used: A universal testing machine from commercial brand Instron - model TTDML (Instron Inc., Canton, MA, USA), a

Table 1 - Distribution of specimens in teardrop loop according to metal alloy, cross-sections and preactivation tested.

\begin{tabular}{ccccc}
\hline \multirow{2}{*}{ Teardrop loops } & $0^{\circ}$ & $\mathbf{4 0 ^ { \circ }}$ & TOTAL \\
\multirow{3}{*}{ Stainless steel } & $0.017 \times 0.025-$ in & 5 & 5 & 10 \\
& $0.019 \times 0.025-$ in & 5 & 5 & 10 \\
\multirow{3}{*}{ Beta-titanium } & $0.017 \times 0.025-$ in & 5 & 5 & 10 \\
& $0.019 \times 0.025-$ in & 5 & 5 & 10 \\
\hline \multirow{3}{*}{ TOTAL } & 20 & 20 & 40 \\
\hline
\end{tabular}

Table 2 - Distribution of the specimens of teardrop loops with helixes accord ing to metal alloy composition, cross-sectional dimension, and preactivation.

\begin{tabular}{ccccc}
\hline \multicolumn{2}{c}{ Teardrop loops with helix } & $0^{\circ}$ & $\mathbf{4 0}^{\circ}$ & TOTAL \\
\multirow{3}{*}{ Stainless steel } & $0.017 \times 0.025-$ in & 5 & 5 & 10 \\
& $0.019 \times 0.025-$ in & 5 & 5 & 10 \\
\multirow{3}{*}{ Beta-titanium } & $0.017 \times 0.025-$ in & 5 & 5 & 10 \\
& $0.019 \times 0.025-$ in & 5 & 5 & 10 \\
\hline \multirow{2}{*}{ TOTAL } & 20 & 20 & 40 \\
\hline
\end{tabular}

Table 3 - Values suggested $(\mathrm{g})$ in the literature to move different groups of teeth in the anterior region.

\begin{tabular}{|c|c|c|c|c|c|c|c|}
\hline & & $\underline{3}$ & $\overline{3}$ & $\underline{21 \mid 12}$ & $\overline{21 \mid 12}$ & $\underline{321 \mid 123}$ & $\overline{321 \mid 123}$ \\
\hline \multirow{2}{*}{ Reitan ${ }^{17}$} & adults & 70 & 67 & 210 & 184 & 350 & 318 \\
\hline & youngs & 113 & 108 & 338 & 296 & 564 & 512 \\
\hline \multicolumn{2}{|c|}{ Jarabak, Fizzell } & $105-170$ & $85-130$ & 320 & 240 & 595 & 455 \\
\hline \multicolumn{2}{|c|}{ Ricketts $^{18}$} & 150 & - & 320 & - & 620 & - \\
\hline \multicolumn{2}{|c|}{ Shimizu ${ }^{20}$} & 150 & 120 & 300 & 240 & 600 & 480 \\
\hline
\end{tabular}




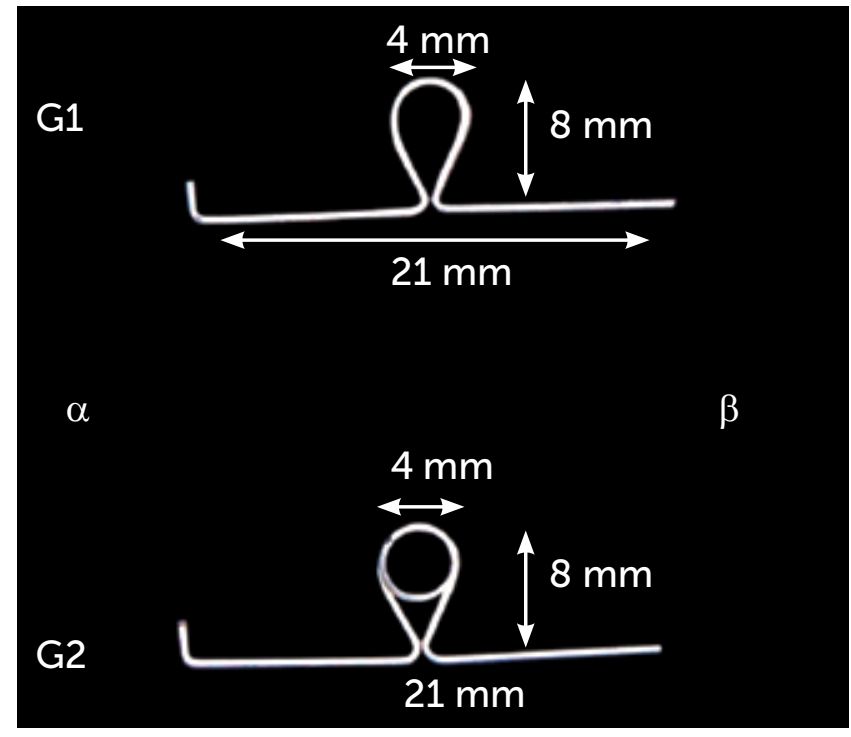

Figure 1 - Configuration of teardrop loop (G1) and teardrop loop with helix (G2) with their respective dimensions

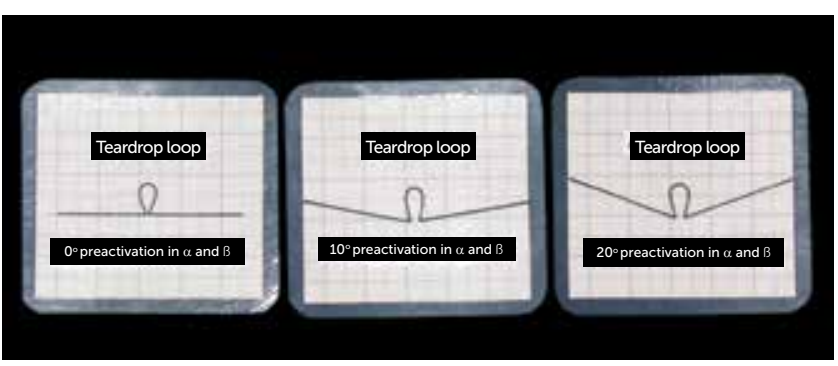

Figure 3 - Templates in graph paper to standardize the fabrication and preac tivation of teardrop loops and teardrop loops with helixes.

transducer of moments and a digital indicator module to extensometry, model TMDE, both from Transdutec (Transdutec Ltda., São Paulo, Brazil) and a comparison dial (Mitutoyo Corporation, Tokyo, Japan) with 10-mm course and 0.01-mm in measurement accuracy, as previously described in other article..$^{25}$

In mechanical test (Fig 5), the loop was symmetrically positioned in a space of $21 \mathrm{~mm}$, between the fixation device and the moment transducer - this position simulates the space inter-brackets from first molar to canine. The loops were submitted to a total activation of 5 $\mathrm{mm}$, and in each $1 \mathrm{~mm}$ of loop activation the test was interrupted and recorded the quantity of force and torsion moment. Consequently it was obtained the L/D and $\mathrm{M} / \mathrm{F}$ ratios. In statistical data analysis, it was performed the analysis of variance considering the level of significance of 5\%, which indicated in what variables or interactions the differences between the averages were statistically significant; and it was supplemented by Tukey's Multiple Comparison Test also in a level of significance

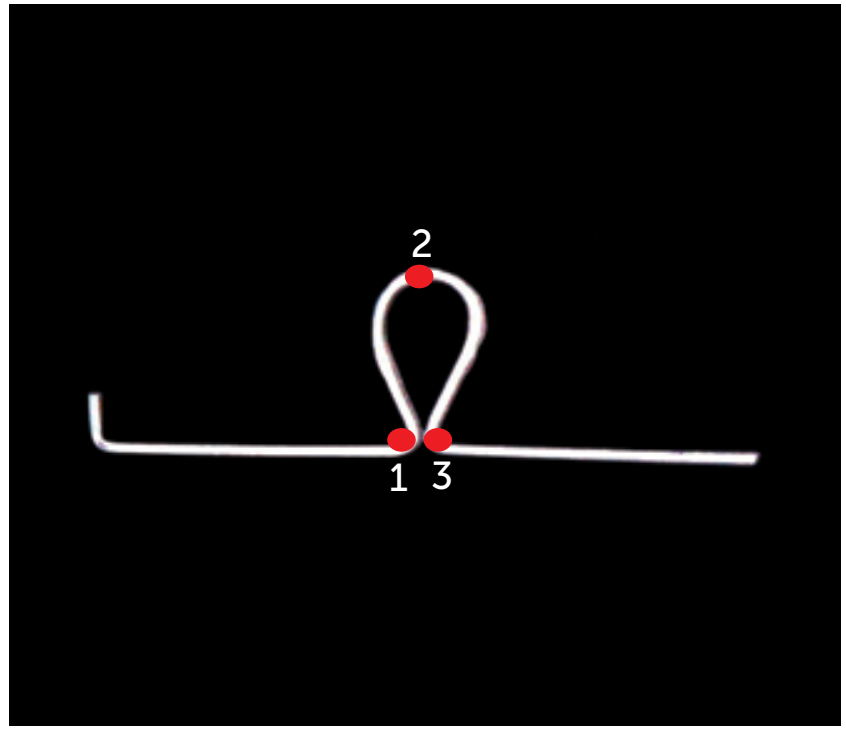

Figure 2 - Places where preactivations were performed in G1 and G2 groups.

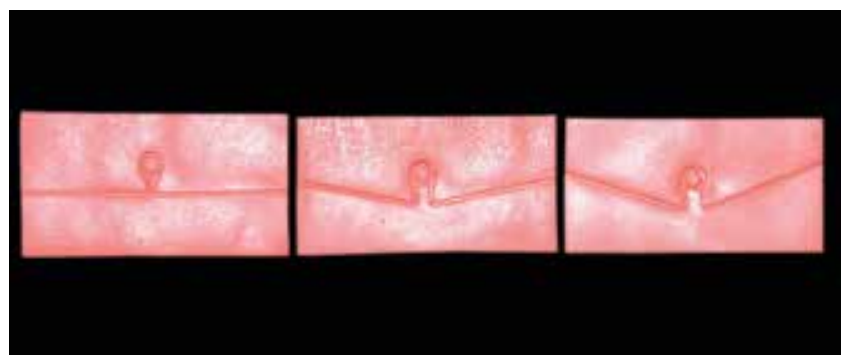

Figure 4 - Templates in vinyl polysiloxane impression material to standardize the fabrication and preactivation of teardrop loops and teardrop loops with helixes

of $5 \%$, indicating what average differed from other. For this purpose it was used the software SAS for Windows V8 (Statistical Analysis System).

\section{RESULTS}

In Table 4 it can be seen that the average and standard deviation for horizontal force (g) produced by teardrop loops when they are submitted from 0 to $5 \mathrm{~mm}$ of activation, according to the interaction between metal alloy, cross-sectional dimension and preactivation. It was possible to observe that, in general way, the horizontal force liberated by teardrop loops suffered greater influence from metal alloy composition, and the stainless steel alloy presented greater magnitude of force than beta-titanium alloy. Relative to the cross-sectional dimension, the $0.019 \times 0.025$-in wire presented higher medium values of horizontal forces. About preactivation bends, their insertion caused an increase of horizontal magnitude of force exerted, regardless of the metal alloy tested. 

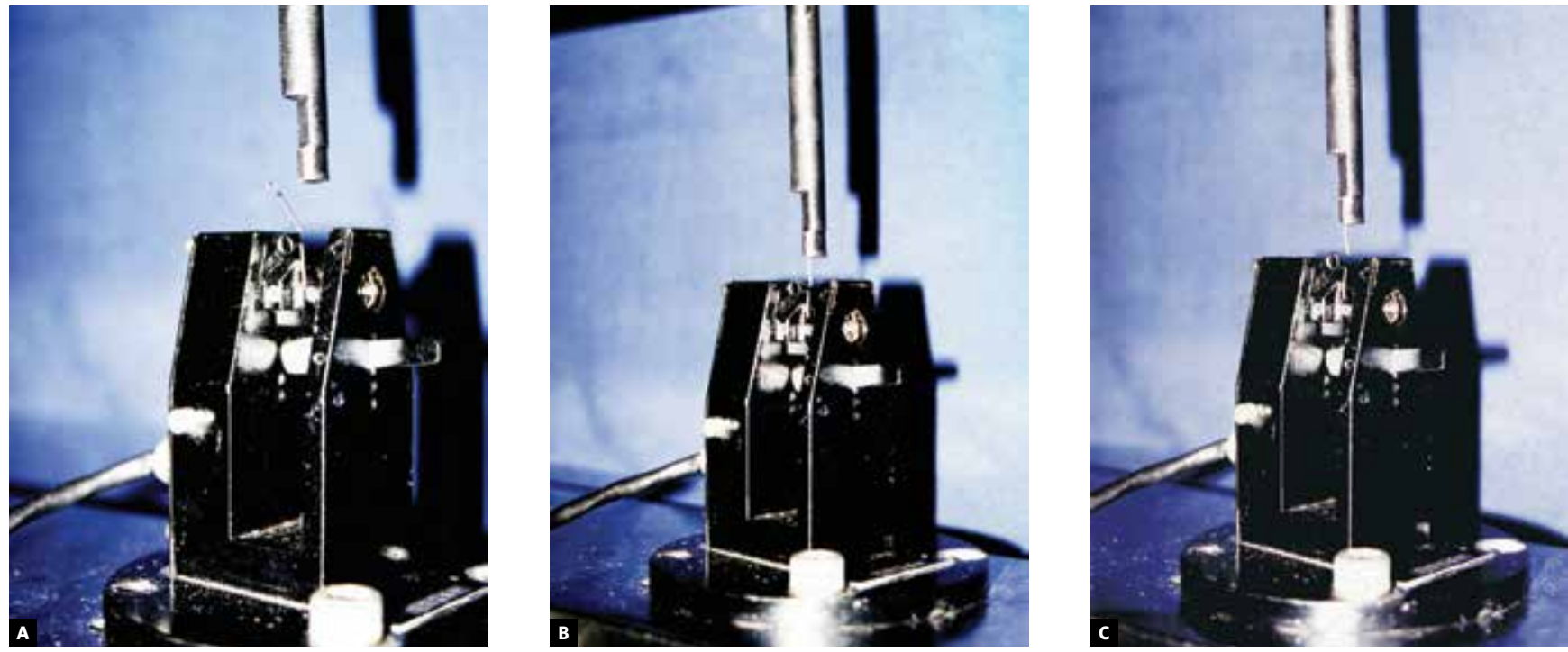

Figure 5 - Mechanical test of specimens: A) preactivated loop fixed in the transducer of moment; B) preactivated loop after insertion on the load cell; C) loop during its activation.

Table 5 presents average and standard deviation to horizontal force (g) produced by teardrop loops with helix. It was possible to observe that, in general, the horizontal force liberated by teardrop loops with helix suffered greater influence of metal alloy composition, and the stainless steel presented higher magnitudes of force than beta-titanium alloy. Relative to the crosssectional dimension and preactivation, loops made with $0.019 \times 0.025$-in wire and preactivated in $40^{\circ}$ presented higher values of horizontal force released.

The Table 6 presents the average and standard deviation to the $\mathrm{M} / \mathrm{F}$ ratio produced by teardrop loops when they are submitted from 0.0 to $5.0 \mathrm{~mm}$ of activation, according to the metal alloy, transversal section and preactivation. It was possible to observe, in general, that the $\mathrm{M} / \mathrm{F}$ ratio produced by teardrop loops suffered greater influence from preactivation, and the loops preactivated in $40^{\circ}$ presented higher ratios than the loops with no preactivation. Related to the metal alloy, the loops made in stainless steel generated a M/F ratio higher than the loops in beta-titanium (except for the activation in $1 \mathrm{~mm}$, where, despite they did not present statistical difference, beta-titanium presented a ratio slightly greater than the stainless steel in the absence of preactivation). In cross-sectional dimension, the $0.017 \times 0.025$-in wires generally produced higher $\mathrm{M} / \mathrm{F}$ ratios than the $0.019 \times 0.025$-in wires, although did not present statistical differences.

The Table 7 presents the average and standard deviation of $\mathrm{M} / \mathrm{F}$ ratio produced by teardrop loops with helix. It was possible to observe, in general, that the $\mathrm{M} / \mathrm{F}$ ratio produced by teardrop loops with helix suffered greater influence of preactivation and preactivated loops in $40^{\circ}$ presented $\mathrm{M} / \mathrm{F}$ ratio higher than loops with no preactivation.

Table 8 presents the average and standard deviation of L/D ratio produced by teardrop loops submitted from 0 to $5 \mathrm{~mm}$ of activation, according to the metal alloy composition, cross-sectional dimension and preactivation. In general way it was observed that the L/D ratio generated by teardrop loops suffered higher influence from metal alloy, and the stainless steel presented higher $\mathrm{L} / \mathrm{D}$ ratio than beta-titanium. Related to the variables at the cross-sectional dimension and preactivation, the loops made with $0.019 \mathrm{x}$ 0.025 -in wire, as well as those with preactivation in $40^{\circ}$ presented higher values in $\mathrm{L} / \mathrm{D}$ ratio.

Table 9 presents averages and standard deviation of the $\mathrm{L} / \mathrm{D}$ ratio produced by teardrop loops with helix. It was verified that, in activations from 1 to $5 \mathrm{~mm}$, the interaction between the metal alloy composition and preactivation was significant, as well as between the metal alloy and cross-sectional dimension. Regardless of the levels of preactivation and cross-sectional dimension, it was found that the medium values of resultant L/D ratio were significantly higher to stainless steel than beta-titanium. In a separate evaluation of the of metal alloy, it was observed that the resultant means were significantly higher when there was preactivation, as well as a $0.019 \times 0.025$-in archwire. 
Table 4 - Means and standard deviations of horizontal forces, in grams, generated by teardrop loops according to the interaction between metal alloy composition, cross-sectional dimension, and preactivation, during the activation of 0 to $5 \mathrm{~mm}$.

\begin{tabular}{|c|c|c|c|c|c|c|c|c|}
\hline \multirow{3}{*}{$\begin{array}{c}\text { Activation } \\
\text { (mm) }\end{array}$} & \multirow{3}{*}{ Metal alloy } & \multirow{3}{*}{ Cross-section } & \multicolumn{4}{|c|}{ Preactivation } & \multicolumn{2}{|c|}{ Total } \\
\hline & & & \multicolumn{2}{|c|}{$0^{\circ}$} & \multicolumn{2}{|c|}{$40^{\circ}$} & \multirow[b]{2}{*}{ Mean } & \multirow[b]{2}{*}{ SD } \\
\hline & & & Mean & SD & Mean & SD & & \\
\hline \multirow{9}{*}{0.0} & \multirow{3}{*}{ Stainless steel } & $0.017 \times 0.025$-in & 0.00 & 0.00 & 14.00 & 4.18 & 7.00 & 7.89 \\
\hline & & $0.019 \times 0.025$-in & 0.00 & 0.00 & 15.00 & 6.12 & 7.50 & 8.90 \\
\hline & & Total & $0.00^{C}$ & 0.00 & $14.50^{\mathrm{A}}$ & 4.97 & 7.25 & 8.19 \\
\hline & \multirow{3}{*}{ Beta-titanium } & $0.017 \times 0.025$-in & 0.00 & 0.00 & 9.00 & 4.18 & 4.50 & 5.50 \\
\hline & & $0.019 \times 0.025$-in & 0.00 & 0.00 & 11.00 & 4.18 & 5.50 & 6.43 \\
\hline & & Total & $0.00^{C}$ & 0.00 & $10.00^{\mathrm{B}}$ & 4.08 & 5.00 & 5.85 \\
\hline & \multirow{3}{*}{ Total } & $0.017 \times 0.025$-in & 0.00 & 0.00 & 11.50 & 4.74 & 5.75 & 6.74 \\
\hline & & $0.019 \times 0.025$-in & 0.00 & 0.00 & 13.00 & 5.37 & 6.50 & 7.63 \\
\hline & & Total & 0.00 & 0.00 & 12.25 & 4.99 & 6.13 & 7.12 \\
\hline \multirow{9}{*}{1.0} & \multirow{3}{*}{ Stainless steel } & $0.017 \times 0.025$-in & 236.00 & 33.62 & 306.00 & 26.08 & $271.00^{b}$ & 46.54 \\
\hline & & $0.019 \times 0.025$-in & 302.00 & 40.25 & 422.00 & 38.99 & $362.00^{\mathrm{a}}$ & 73.45 \\
\hline & & Total & $269.00^{\mathrm{B}}$ & 49.32 & $364.00^{\mathrm{A}}$ & 68.67 & 316.50 & 75.90 \\
\hline & \multirow{3}{*}{ Beta-titanium } & $0.017 \times 0.025$-in & 94.00 & 15.17 & 143.00 & 8.37 & $118.50^{d}$ & 28.29 \\
\hline & & $0.019 \times 0.025$-in & 156.00 & 30.50 & 184.00 & 8.94 & $170.00^{c}$ & 25.82 \\
\hline & & Total & $125.00^{\mathrm{D}}$ & 39.79 & $163.50^{C}$ & 23.10 & 144.25 & 37.32 \\
\hline & & $0.017 \times 0.025$-in & 165.00 & 78.78 & 224.50 & 87.83 & 194.75 & 86.75 \\
\hline & Total & $0.019 \times 0.025$-in & 229.00 & 83.99 & 303.00 & 128.24 & 266.00 & 112.13 \\
\hline & & Total & 197.00 & 85.78 & 263.75 & 114.30 & 230.38 & 105.32 \\
\hline & & $0.017 \times 0.025$-in & $452.00^{C}$ & 47.64 & $581.00^{\mathrm{B}}$ & 22.47 & 516.50 & 76.52 \\
\hline & Stainless steel & $0.019 \times 0.025$-in & $596.00^{\mathrm{B}}$ & 45.61 & $832.00^{A}$ & 37.01 & 714.00 & 130.40 \\
\hline & & Total & 524.00 & 87.71 & 706.50 & 135.40 & 615.25 & 145.24 \\
\hline & & $0.017 \times 0.025$-in & $198.00^{\mathrm{F}}$ & 19.56 & $262.00^{\mathrm{EF}}$ & 21.68 & 230.00 & 38.94 \\
\hline 2.0 & Beta-titanium & $0.019 \times 0.025$-in & $313.00^{\mathrm{DE}}$ & 27.75 & $358.00^{\mathrm{D}}$ & 19.24 & 335.50 & 32.70 \\
\hline & & Total & 255.50 & 64.70 & 310.00 & 54.16 & 282.75 & 64.45 \\
\hline & & $0.017 \times 0.025$-in & 325.00 & 138.20 & 421.50 & 169.41 & 373.25 & 158.41 \\
\hline & Total & $0.019 \times 0.025$-in & 454.50 & 153.34 & 595.00 & 251.36 & 524.75 & 215.09 \\
\hline & & Total & 389.75 & 156.84 & 508.25 & 226.82 & 449.00 & 201.61 \\
\hline & & $0.017 \times 0.025$-in & $622.00^{c}$ & 49.07 & $822.00^{\mathrm{B}}$ & 19.24 & 722.00 & 111.11 \\
\hline & Stainless steel & $0.019 \times 0.025$-in & $837.00^{\mathrm{B}}$ & 33.84 & $1176.00^{A}$ & 18.17 & 1006.50 & 180.49 \\
\hline & & Total & 729.50 & 120.08 & 999.00 & 187.41 & 864.25 & 206.35 \\
\hline & & $0.017 \times 0.025$-in & $298.00^{G}$ & 22.53 & $390.00^{F}$ & 24.49 & 344.00 & 53.32 \\
\hline 3.0 & Beta-titanium & $0.019 \times 0.025$-in & $454.00^{\mathrm{E}}$ & 31.30 & $537.00^{\circ}$ & 29.50 & 495.50 & 52.31 \\
\hline & & Total & 376.00 & 86.15 & 463.50 & 81.58 & 419.75 & 93.18 \\
\hline & & $0.017 \times 0.025$-in & 460.00 & 174.52 & 606.00 & 228.63 & 533.00 & 211.65 \\
\hline & Total & $0.019 \times 0.025$-in & 645.50 & 204.18 & 856.50 & 337.57 & 751.00 & 292.31 \\
\hline & & Total & 552.75 & 207.92 & 731.25 & 308.63 & 642.00 & 275.02 \\
\hline & & $0.017 \times 0.025$-in & $761.00^{\mathrm{C}}$ & 47.22 & $1016.00^{\mathrm{B}}$ & 15.17 & 888.50 & 138.40 \\
\hline & Stainless steel & $0.019 \times 0.025$-in & $1037.00^{\mathrm{B}}$ & 29.07 & $1432.00^{A}$ & 16.43 & 1234.50 & 209.37 \\
\hline & & Total & 899.00 & 150.09 & 1224.00 & 219.76 & 1061.50 & 247.67 \\
\hline & & $0.017 \times 0.025$-in & $387.00^{F}$ & 22.25 & $511.00^{\mathrm{E}}$ & 27.02 & 449.00 & 69.39 \\
\hline 4.0 & Beta-titanium & $0.019 \times 0.025$-in & $577.00^{\circ}$ & 30.12 & $704.00^{\mathrm{C}}$ & 37.82 & 640.50 & 74.29 \\
\hline & & Total & 482.00 & 103.20 & 607.50 & 106.33 & 544.75 & 120.61 \\
\hline & & $0.017 \times 0.025$-in & 574.00 & 200.16 & 763.50 & 266.96 & 668.75 & 249.37 \\
\hline & Total & $0.019 \times 0.025$-in & 807.00 & 244.04 & 1068.00 & 384.67 & 937.50 & 340.93 \\
\hline & & Total & 690.50 & 247.94 & 915.75 & 358.12 & 803.13 & 324.72 \\
\hline & & $0.017 \times 0.025$-in & $891.00^{C}$ & 54.59 & $1176.00^{\mathrm{B}}$ & 16.73 & 1033.50 & 154.96 \\
\hline & Stainless steel & $0.019 \times 0.025$-in & $1215.00^{\mathrm{B}}$ & 36.40 & $1633.00^{A}$ & 15.65 & 1424.00 & 221.88 \\
\hline & & Total & 1053.00 & 176.28 & 1404.50 & 241.34 & 1228.75 & 273.54 \\
\hline & & $0.017 \times 0.025$-in & $468.00^{\mathrm{E}}$ & 23.61 & $622.00^{\mathrm{D}}$ & 31.34 & 545.00 & 85.28 \\
\hline 5.0 & Beta-titanium & $0.019 \times 0.025$-in & $685.00^{\mathrm{D}}$ & 28.50 & $848.00^{\mathrm{C}}$ & 41.47 & 766.50 & 92.23 \\
\hline & & Total & 576.50 & 117.00 & 735.00 & 124.05 & 655.75 & 142.78 \\
\hline & & $0.017 \times 0.025$-in & 679.50 & 226.44 & 899.00 & 292.94 & 789.25 & 278.60 \\
\hline & Total & $0.019 \times 0.025$-in & 950.00 & 281.03 & 1240.50 & 414.79 & 1095.25 & 375.65 \\
\hline & & Total & 814.75 & 284.52 & 1069.75 & 390.94 & 942.25 & 361.35 \\
\hline
\end{tabular}

For each activation, means followed by distinct letters differ significantly by means of the analysis of variance (lower case and uppercase are used for independent comparisons), supplemented by Tukey's Multiple Comparison Test, in a level of significance of 5\%. 
Table 5 - Means and standard deviations of horizontal forces, in grams, generated by teardrop loops with helix, according to the interaction between metal alloy composition, cross-section, and preactivation, during the activation of $0 \mathrm{~mm}$ to $5 \mathrm{~mm}$.

\begin{tabular}{|c|c|c|c|c|c|c|c|c|}
\hline \multirow{3}{*}{$\begin{array}{c}\text { Activation } \\
(\mathrm{mm})\end{array}$} & \multirow{3}{*}{ Metal alloy } & \multirow{3}{*}{ Cross-section } & \multicolumn{4}{|c|}{ Preactivation } & \multirow{2}{*}{\multicolumn{2}{|c|}{ Total }} \\
\hline & & & \multicolumn{2}{|c|}{$0^{\circ}$} & \multicolumn{2}{|c|}{$40^{\circ}$} & & \\
\hline & & & Mean & SD & Mean & SD & Mean & SD \\
\hline \multirow{9}{*}{0.0} & \multirow{3}{*}{ Stainless steel } & $0.017 \times 0.025$-in & 0.00 & 0.00 & 16.00 & 6.52 & 8.00 & 9.49 \\
\hline & & $0.019 \times 0.025$-in & 0.00 & 0.00 & 17.00 & 4.47 & 8.50 & 9.44 \\
\hline & & Total & $0.00^{C}$ & 0.00 & $16.50^{\mathrm{A}}$ & 5.30 & 8.25 & 9.22 \\
\hline & \multirow{3}{*}{ Beta-titanium } & $0.017 \times 0.025$-in & 0.00 & 0.00 & 7.00 & 2.74 & 3.50 & 4.12 \\
\hline & & $0.019 \times 0.025$-in & 0.00 & 0.00 & 7.00 & 2.74 & 3.50 & 4.12 \\
\hline & & Total & $0.00^{C}$ & 0.00 & $7.00^{\mathrm{B}}$ & 2.58 & 3.50 & 4.01 \\
\hline & \multirow{3}{*}{ Total } & $0.017 \times 0.025$-in & 0.00 & 0.00 & 11.50 & 6.69 & 5.75 & 7.48 \\
\hline & & $0.019 \times 0.025$-in & 0.00 & 0.00 & 12.00 & 6.32 & 6.00 & 7.54 \\
\hline & & Total & 0.00 & 0.00 & 11.75 & 6.34 & 5.88 & 7.42 \\
\hline \multirow{9}{*}{1.0} & \multirow{3}{*}{ Stainless steel } & $0.017 \times 0.025$-in & 155.00 & 13.23 & 203.00 & 26.83 & $179.00^{b}$ & 32.21 \\
\hline & & $0.019 \times 0.025$-in & 212.00 & 16.81 & 272.00 & 17.89 & $242.00^{a}$ & 35.61 \\
\hline & & Total & $183.50^{\mathrm{B}}$ & 33.25 & $237.50^{\mathrm{A}}$ & 42.25 & 210.50 & 46.22 \\
\hline & \multirow{3}{*}{ Beta-titanium } & $0.017 \times 0.025$-in & 69.00 & 4.18 & 94.00 & 8.22 & $81.50^{d}$ & 14.54 \\
\hline & & $0.019 \times 0.025$-in & 87.00 & 8.37 & 117.00 & 4.47 & $102.00^{c}$ & 17.03 \\
\hline & & Total & $78.00^{\mathrm{D}}$ & 11.35 & $105.50^{C}$ & 13.63 & 91.75 & 18.66 \\
\hline & \multirow{3}{*}{ Total } & $0.017 \times 0.025$-in & 112.00 & 46.26 & 148.50 & 60.42 & 130.25 & 55.62 \\
\hline & & $0.019 \times 0.025$-in & 149.50 & 67.06 & 194.50 & 82.61 & 172.00 & 76.78 \\
\hline & & Total & 130.75 & 59.28 & 171.50 & 74.29 & 151.13 & 69.47 \\
\hline \multirow{9}{*}{2.0} & & $0.017 \times 0.025$-in & 326.00 & 16.36 & 396.00 & 36.47 & $361.00^{b}$ & 45.51 \\
\hline & Stainless steel & $0.019 \times 0.025$-in & 446.00 & 25.10 & 535.00 & 34.28 & $490.50^{a}$ & 54.80 \\
\hline & & Total & $386.00^{\mathrm{B}}$ & 66.32 & $465.50^{\mathrm{A}}$ & 80.50 & 425.75 & 82.56 \\
\hline & & $0.017 \times 0.025$-in & 150.00 & 6.12 & 188.00 & 13.51 & $169.00^{d}$ & 22.34 \\
\hline & Beta-titanium & $0.019 \times 0.025$-in & 178.00 & 18.91 & 224.00 & 11.94 & $201.00^{c}$ & 28.46 \\
\hline & & Total & $164.00^{D}$ & 19.83 & $206.00^{C}$ & 22.46 & 185.00 & 29.82 \\
\hline & & $0.017 \times 0.025$-in & 238.00 & 93.49 & 292.00 & 112.65 & 265.00 & 104.49 \\
\hline & Total & $0.019 \times 0.025$-in & 312.00 & 142.79 & 379.50 & 165.69 & 345.75 & 154.47 \\
\hline & & Total & 275.00 & 123.45 & 335.75 & 145.02 & 305.38 & 136.44 \\
\hline & & $0.017 \times 0.025$-in & 502.00 & 21.97 & 595.00 & 43.01 & $548.50^{b}$ & 58.64 \\
\hline & Stainless steel & $0.019 \times 0.025$-in & 660.00 & 39.37 & 802.00 & 44.38 & $731.00^{a}$ & 84.65 \\
\hline & & Total & $581.00^{\mathrm{B}}$ & 88.53 & $698.50^{A}$ & 116.62 & 639.75 & 117.42 \\
\hline & & $0.017 \times 0.025$-in & 234.00 & 15.57 & 282.00 & 14.40 & $258.00^{d}$ & 28.98 \\
\hline 3.0 & Beta-titanium & $0.019 \times 0.025$-in & 277.00 & 25.40 & 326.00 & 21.04 & $301.50^{c}$ & 33.92 \\
\hline & & Total & $255.50^{\mathrm{D}}$ & 30.13 & $304.00^{C}$ & 28.75 & 279.75 & 37.96 \\
\hline & & $0.017 \times 0.025$-in & 368.00 & 142.38 & 438.50 & 167.71 & 403.25 & 155.68 \\
\hline & Total & $0.019 \times 0.025$-in & 468.50 & 204.26 & 564.00 & 253.00 & 516.25 & 229.09 \\
\hline & & Total & 418.25 & 178.95 & 501.25 & 218.61 & 459.75 & 201.62 \\
\hline & & $0.017 \times 0.025$-in & 660.00 & 25.25 & 780.00 & 46.37 & $720.00^{b}$ & 72.38 \\
\hline & Stainless steel & $0.019 \times 0.025$-in & 857.00 & 47.38 & 1053.00 & 54.50 & $955.00^{a}$ & 113.97 \\
\hline & & Total & $758.50^{\mathrm{B}}$ & 109.82 & $916.50^{A}$ & 151.59 & 837.50 & 152.21 \\
\hline & & $0.017 \times 0.025$-in & 330.00 & 20.00 & 378.00 & 17.54 & $354.00^{d}$ & 30.89 \\
\hline 4.0 & Beta-titanium & $0.019 \times 0.025$-in & 386.00 & 35.43 & 439.00 & 27.48 & $412.50^{c}$ & 40.91 \\
\hline & & Total & $358.00^{\mathrm{D}}$ & 40.08 & $408.50^{C}$ & 38.81 & 383.25 & 46.32 \\
\hline & & $0.017 \times 0.025$-in & 495.00 & 175.25 & 579.00 & 214.43 & 537.00 & 195.41 \\
\hline & Total & $0.019 \times 0.025$-in & 621.50 & 251.35 & 746.00 & 326.15 & 683.75 & 290.51 \\
\hline & & Total & 558.25 & 220.65 & 662.50 & 281.97 & 610.38 & 255.42 \\
\hline & & $0.017 \times 0.025$-in & 816.00 & 33.05 & 954.00 & 45.06 & $885.00^{b}$ & 81.72 \\
\hline & Stainless steel & $0.019 \times 0.025$-in & 1044.00 & 60.77 & 1276.00 & 68.04 & $1160.00^{a}$ & 136.57 \\
\hline & & Total & $930.00^{\mathrm{B}}$ & 128.71 & $1115.00^{\mathrm{A}}$ & 178.22 & 1022.50 & 178.60 \\
\hline & & $0.017 \times 0.025$-in & 424.00 & 31.50 & 479.00 & 22.47 & $451.50^{d}$ & 38.81 \\
\hline 5.0 & Beta-titanium & $0.019 \times 0.025$-in & 495.00 & 40.00 & 558.00 & 34.93 & $526.50^{c}$ & 48.54 \\
\hline & & Total & $459.50^{D}$ & 50.52 & $518.50^{C}$ & 50.00 & 489.00 & 57.53 \\
\hline & & $0.017 \times 0.025$-in & 620.00 & 208.83 & 716.50 & 252.59 & 668.25 & 230.93 \\
\hline & Total & $0.019 \times 0.025$-in & 769.50 & 293.38 & 917.00 & 381.84 & 843.25 & 339.94 \\
\hline & & Total & 694.75 & 259.45 & 816.75 & 331.46 & 755.75 & 300.22 \\
\hline
\end{tabular}

For each activation, means followed by distinct letters differ significantly by means of the analysis of variance (lower case and uppercase are used for independent comparisons), supplemented by Tukey's Multiple Comparison Test, in a level of significance of 5\%. 
Table 6 - Means and standard deviations of moment/force (M/F) ratio, in mm, generated by teardrop loops according to the interaction between metal alloy composition, cross-section, and preactivation during the activation of $0 \mathrm{~mm}$ to $5 \mathrm{~mm}$.

\begin{tabular}{|c|c|c|c|c|c|c|c|c|}
\hline \multirow{3}{*}{$\begin{array}{c}\text { Activation } \\
\text { (mm) }\end{array}$} & \multirow{3}{*}{ Metal alloy } & \multirow{3}{*}{ Cross-section } & \multicolumn{4}{|c|}{ Preactivation } & \multicolumn{2}{|c|}{ Total } \\
\hline & & & \multicolumn{2}{|c|}{$0^{\circ}$} & \multicolumn{2}{|c|}{$40^{\circ}$} & \multirow[b]{2}{*}{ Mean } & \multirow[b]{2}{*}{ SD } \\
\hline & & & Mean & SD & Mean & SD & & \\
\hline \multirow{9}{*}{0.0} & \multirow{3}{*}{ Stainless steel } & $0.017 \times 0.025$-in & 0.00 & 0.00 & 107.13 & 30.15 & 53.57 & 59.93 \\
\hline & & $0.019 \times 0.025$-in & 0.00 & 0.00 & 128.68 & 56.30 & 64.34 & 77.51 \\
\hline & & Total & $0.00^{C}$ & 0.00 & $117.91^{\mathrm{A}}$ & 44.06 & 58.95 & 67.66 \\
\hline & \multirow{3}{*}{ Beta-titanium } & $0.017 \times 0.025$-in & 0.00 & 0.00 & 67.20 & 32.65 & 33.60 & 41.57 \\
\hline & & $0.019 \times 0.025$-in & 0.00 & 0.00 & 63.80 & 33.59 & 31.90 & 40.40 \\
\hline & & Total & $0.00^{c}$ & 0.00 & $65.50^{\mathrm{B}}$ & 31.28 & 32.75 & 39.90 \\
\hline & \multirow{3}{*}{ Total } & $0.017 \times 0.025$-in & 0.00 & 0.00 & 87.17 & 36.34 & 43.58 & 51.23 \\
\hline & & $0.019 \times 0.025$-in & 0.00 & 0.00 & 96.24 & 55.49 & 48.12 & 62.42 \\
\hline & & Total & 0.00 & 0.00 & 91.70 & 45.89 & 45.85 & 56.41 \\
\hline \multirow{9}{*}{1.0} & \multirow{3}{*}{ Stainless steel } & $0.017 \times 0.025$-in & 1.42 & 0.07 & 5.29 & 0.43 & 3.35 & 2.06 \\
\hline & & $0.019 \times 0.025$-in & 1.31 & 0.16 & 4.79 & 0.66 & 3.05 & 1.89 \\
\hline & & Total & $1.36^{c}$ & 0.13 & $5.04^{A}$ & 0.59 & 3.20 & 1.93 \\
\hline & \multirow{3}{*}{ Beta-titanium } & $0.017 \times 0.025$-in & 1.55 & 0.32 & 4.33 & 0.41 & 2.94 & 1.50 \\
\hline & & $0.019 \times 0.025$-in & 1.21 & 0.13 & 4.22 & 0.11 & 2.71 & 1.59 \\
\hline & & Total & $1.38^{\mathrm{C}}$ & 0.29 & $4.27^{\mathrm{B}}$ & 0.29 & 2.83 & 1.51 \\
\hline & & $0.017 \times 0.025$-in & 1.48 & 0.23 & 4.81 & 0.64 & 3.15 & 1.77 \\
\hline & Total & $0.019 \times 0.025$-in & 1.26 & 0.15 & 4.50 & 0.54 & 2.88 & 1.71 \\
\hline & & Total & 1.37 & 0.22 & 4.66 & 0.60 & 3.01 & 1.72 \\
\hline & & $0.017 \times 0.025$-in & 1.58 & 0.15 & 3.00 & 0.17 & 2.29 & 0.76 \\
\hline & Stainless steel & $0.019 \times 0.025$-in & 1.64 & 0.25 & 2.74 & 0.26 & 2.19 & 0.63 \\
\hline & & Total & 1.61 & 0.20 & 2.87 & 0.25 & $2.24^{a}$ & 0.68 \\
\hline & & $0.017 \times 0.025$-in & 1.27 & 0.18 & 2.59 & 0.33 & 1.93 & 0.74 \\
\hline 2.0 & Beta-titanium & $0.019 \times 0.025$-in & 1.22 & 0.08 & 2.45 & 0.11 & 1.84 & 0.65 \\
\hline & & Total & 1.25 & 0.14 & 2.52 & 0.24 & $1.89^{b}$ & 0.68 \\
\hline & & $0.017 \times 0.025$-in & 1.43 & 0.23 & 2.80 & 0.33 & 2.11 & 0.75 \\
\hline & Total & $0.019 \times 0.025$-in & 1.43 & 0.28 & 2.60 & 0.24 & 2.01 & 0.65 \\
\hline & & Total & $1.43^{B}$ & 0.25 & $2.70^{A}$ & 0.30 & 2.06 & 0.70 \\
\hline & & $0.017 \times 0.025$-in & 1.62 & 0.17 & 2.22 & 0.13 & 1.92 & 0.35 \\
\hline & Stainless steel & $0.019 \times 0.025$-in & 1.67 & 0.22 & 2.06 & 0.13 & 1.86 & 0.27 \\
\hline & & Total & 1.64 & 0.19 & 2.14 & 0.15 & $1.89^{a}$ & 0.30 \\
\hline & & $0.017 \times 0.025$-in & 1.27 & 0.15 & 1.86 & 0.21 & 1.56 & 0.35 \\
\hline 3.0 & Beta-titanium & $0.019 \times 0.025$-in & 1.24 & 0.06 & 1.77 & 0.06 & 1.51 & 0.28 \\
\hline & & Total & 1.26 & 0.11 & 1.81 & 0.15 & $1.53^{b}$ & 0.31 \\
\hline & & $0.017 \times 0.025$-in & 1.44 & 0.24 & 2.04 & 0.25 & 1.74 & 0.39 \\
\hline & Total & $0.019 \times 0.025$-in & 1.46 & 0.27 & 1.91 & 0.18 & 1.68 & 0.32 \\
\hline & & Total & $1.45^{\mathrm{B}}$ & 0.25 & $1.98^{A}$ & 0.22 & 1.71 & 0.35 \\
\hline & & $0.017 \times 0.025$-in & 1.61 & 0.17 & 1.84 & 0.10 & 1.73 & 0.18 \\
\hline & Stainless steel & $0.019 \times 0.025$-in & 1.67 & 0.20 & 1.73 & 0.11 & 1.70 & 0.15 \\
\hline & & Total & 1.64 & 0.18 & 1.79 & 0.11 & $1.72^{a}$ & 0.16 \\
\hline & & $0.017 \times 0.025$-in & 1.24 & 0.11 & 1.49 & 0.17 & 1.37 & 0.19 \\
\hline 4.0 & Beta-titanium & $0.019 \times 0.025$-in & 1.24 & 0.07 & 1.42 & 0.05 & 1.33 & 0.11 \\
\hline & & Total & 1.24 & 0.09 & 1.46 & 0.12 & $1.35^{b}$ & 0.15 \\
\hline & & $0.017 \times 0.025$-in & 1.43 & 0.24 & 1.67 & 0.23 & 1.55 & 0.26 \\
\hline & Total & $0.019 \times 0.025$-in & 1.45 & 0.27 & 1.58 & 0.18 & 1.52 & 0.23 \\
\hline & & Total & $1.44^{\mathrm{B}}$ & 0.25 & $1.62^{\mathrm{A}}$ & 0.21 & 1.53 & 0.24 \\
\hline & & $0.017 \times 0.025$-in & 1.58 & 0.15 & 1.62 & 0.08 & 1.60 & 0.12 \\
\hline & Stainless steel & $0.019 \times 0.025$-in & 1.64 & 0.19 & 1.53 & 0.08 & 1.59 & 0.15 \\
\hline & & Total & 1.61 & 0.17 & 1.58 & 0.09 & $1.59^{a}$ & 0.13 \\
\hline & & $0.017 \times 0.025$-in & 1.22 & 0.09 & 1.25 & 0.14 & 1.23 & 0.11 \\
\hline 5.0 & Beta-titanium & $0.019 \times 0.025$-in & 1.22 & 0.08 & 1.22 & 0.03 & 1.22 & 0.06 \\
\hline & & Total & 1.22 & 0.08 & 1.24 & 0.10 & $1.23^{b}$ & 0.09 \\
\hline & & $0.017 \times 0.025$-in & 1.40 & 0.23 & 1.44 & 0.22 & 1.42 & 0.22 \\
\hline & Total & $0.019 \times 0.025$-in & 1.43 & 0.26 & 1.38 & 0.18 & 1.40 & 0.22 \\
\hline & & Total & 1.41 & 0.24 & 1.41 & 0.20 & 1.41 & 0.22 \\
\hline
\end{tabular}

For each activation, means followed by distinct letters differ significantly by means of the analysis of variance (lower case and uppercase are used for independent comparisons), supplemented by Tukey's Multiple Comparison Test, in a level of significance of 5\%. 
Table 7 - Means and standard deviations of moment/force (M/F) ratio, in mm, generated by teardrop loop with helix according to the interaction between metal alloy composition, cross-section, and preactivation during the activation of $0 \mathrm{~mm}$ to $5 \mathrm{~mm}$.

\begin{tabular}{|c|c|c|c|c|c|c|c|c|}
\hline \multirow{3}{*}{$\begin{array}{c}\text { Activation } \\
(\mathrm{mm})\end{array}$} & \multirow{3}{*}{ Metal alloy } & \multirow{3}{*}{ Cross -section } & \multicolumn{4}{|c|}{ Preactivation } & \multicolumn{2}{|c|}{ Total } \\
\hline & & & \multicolumn{2}{|c|}{$0^{\circ}$} & \multicolumn{2}{|c|}{$40^{\circ}$} & \multirow[b]{2}{*}{ Mean } & \multirow[b]{2}{*}{ SD } \\
\hline & & & Mean & SD & Mean & SD & & \\
\hline \multirow{9}{*}{0.0} & \multirow{3}{*}{ Stainless steel } & $0.017 \times 0.025$-in & 0.00 & 0.00 & 84.50 & 42.39 & 42.25 & 52.75 \\
\hline & & $0.019 \times 0.025$-in & 0.00 & 0.00 & 102.10 & 34.44 & 51.05 & 58.50 \\
\hline & & Total & 0.00 & 0.00 & 93.30 & 37.57 & 46.65 & 54.40 \\
\hline & \multirow{3}{*}{ Beta-titanium } & $0.017 \times 0.025$-in & 0.00 & 0.00 & 56.60 & 15.32 & 28.30 & 31.53 \\
\hline & & $0.019 \times 0.025$-in & 0.00 & 0.00 & 88.40 & 34.36 & 44.20 & 51.92 \\
\hline & & Total & 0.00 & 0.00 & 72.50 & 30.17 & 36.25 & 42.59 \\
\hline & \multirow{3}{*}{ Total } & $0.017 \times 0.025$-in & 0.00 & 0.00 & 70.55 & 33.46 & 35.28 & 42.90 \\
\hline & & $0.019 \times 0.025$-in & 0.00 & 0.00 & 95.25 & 33.23 & 47.63 & 53.95 \\
\hline & & Total & $0.00^{b}$ & 0.00 & $82.90^{\mathrm{a}}$ & 34.84 & 41.45 & 48.51 \\
\hline \multirow{9}{*}{1.0} & \multirow{3}{*}{ Stainless steel } & $0.017 \times 0.025$-in & 2.32 & 0.18 & 7.43 & 1.16 & 4.88 & 2.80 \\
\hline & & $0.019 \times 0.025$-in & 3.05 & 0.48 & 7.56 & 0.99 & 5.31 & 2.48 \\
\hline & & Total & 2.69 & 0.51 & 7.50 & 1.02 & $5.09^{a}$ & 2.59 \\
\hline & \multirow{3}{*}{ Beta-titanium } & $0.017 \times 0.025$-in & 1.78 & 0.28 & 5.55 & 0.82 & 3.66 & 2.07 \\
\hline & & $0.019 \times 0.025$-in & 2.12 & 0.23 & 6.57 & 1.15 & 4.34 & 2.47 \\
\hline & & Total & 1.95 & 0.30 & 6.06 & 1.08 & $4.00^{b}$ & 2.25 \\
\hline & & $0.017 \times 0.025$-in & 2.05 & 0.36 & 6.49 & 1.37 & $4.27^{a}$ & 2.48 \\
\hline & Total & $0.019 \times 0.025$-in & 2.59 & 0.61 & 7.06 & 1.14 & $4.82^{\beta}$ & 2.46 \\
\hline & & Total & $2.32^{b}$ & 0.56 & $6.78^{a}$ & 1.26 & 4.55 & 2.45 \\
\hline & & $0.017 \times 0.025$-in & 2.35 & 0.24 & 4.33 & 0.52 & 3.34 & 1.11 \\
\hline & Stainless steel & $0.019 \times 0.025$-in & 2.74 & 0.32 & 4.34 & 0.50 & 3.54 & 0.93 \\
\hline & & Total & 2.54 & 0.33 & 4.33 & 0.48 & $3.44^{\mathrm{a}}$ & 1.00 \\
\hline & & $0.017 \times 0.025$-in & 1.68 & 0.22 & 3.40 & 0.53 & 2.54 & 0.99 \\
\hline 2.0 & Beta-titanium & $0.019 \times 0.025$-in & 2.05 & 0.26 & 4.12 & 0.75 & 3.09 & 1.21 \\
\hline & & Total & 1.86 & 0.30 & 3.76 & 0.72 & $2.81^{b}$ & 1.11 \\
\hline & & $0.017 \times 0.025$-in & 2.01 & 0.42 & 3.87 & 0.70 & $2.94^{\alpha}$ & 1.10 \\
\hline & Total & $0.019 \times 0.025$-in & 2.39 & 0.45 & 4.23 & 0.61 & $3.31^{\beta}$ & 1.08 \\
\hline & & Total & $2.20^{b}$ & 0.47 & $4.05^{a}$ & 0.66 & 3.13 & 1.09 \\
\hline & & $0.017 \times 0.025$-in & 2.15 & 0.12 & 3.17 & 0.33 & 2.66 & 0.59 \\
\hline & Stainless steel & $0.019 \times 0.025$-in & 2.55 & 0.26 & 3.18 & 0.37 & 2.87 & 0.45 \\
\hline & & Total & 2.35 & 0.28 & 3.17 & 0.33 & $2.76^{a}$ & 0.52 \\
\hline & & $0.017 \times 0.025$-in & 1.61 & 0.18 & 2.56 & 0.34 & 2.09 & 0.56 \\
\hline 3.0 & Beta-titanium & $0.019 \times 0.025$-in & 1.97 & 0.21 & 3.18 & 0.57 & 2.57 & 0.75 \\
\hline & & Total & 1.79 & 0.26 & 2.87 & 0.55 & $2.33^{b}$ & 0.69 \\
\hline & & $0.017 \times 0.025$-in & 1.88 & 0.32 & 2.87 & 0.45 & $2.37^{a}$ & 0.63 \\
\hline & Total & $0.019 \times 0.025$-in & 2.26 & 0.38 & 3.18 & 0.45 & $2.72^{\beta}$ & 0.62 \\
\hline & & Total & $2.07^{b}$ & 0.39 & $3.02^{a}$ & 0.47 & 2.55 & 0.64 \\
\hline & & $0.017 \times 0.025$-in & 2.00 & 0.13 & 2.56 & 0.24 & 2.28 & 0.34 \\
\hline & Stainless steel & $0.019 \times 0.025$-in & 2.35 & 0.18 & 2.55 & 0.33 & 2.45 & 0.27 \\
\hline & & Total & 2.17 & 0.23 & 2.55 & 0.27 & $2.36^{a}$ & 0.31 \\
\hline & & $0.017 \times 0.025$-in & 1.47 & 0.12 & 2.08 & 0.26 & 1.78 & 0.37 \\
\hline 4.0 & Beta-titanium & $0.019 \times 0.025$-in & 1.82 & 0.19 & 2.56 & 0.45 & 2.19 & 0.51 \\
\hline & & Total & 1.65 & 0.24 & 2.32 & 0.43 & $1.98^{b}$ & 0.48 \\
\hline & & $0.017 \times 0.025$-in & 1.74 & 0.30 & 2.32 & 0.34 & $2.03^{a}$ & 0.43 \\
\hline & Total & $0.019 \times 0.025$-in & 2.08 & 0.33 & 2.55 & 0.37 & $2.32^{\beta}$ & 0.42 \\
\hline & & Total & $1.91^{b}$ & 0.35 & $2.44^{a}$ & 0.37 & 2.17 & 0.45 \\
\hline & & $0.017 \times 0.025$-in & 1.86 & 0.13 & 2.14 & 0.16 & 2.00 & 0.20 \\
\hline & Stainless steel & $0.019 \times 0.025$-in & 2.17 & 0.14 & 2.16 & 0.30 & 2.17 & 0.22 \\
\hline & & Total & 2.02 & 0.21 & 2.15 & 0.23 & $2.08^{a}$ & 0.22 \\
\hline & & $0.017 \times 0.025$-in & 1.36 & 0.07 & 1.72 & 0.20 & 1.54 & 0.24 \\
\hline 5.0 & Beta-titanium & $0.019 \times 0.025$-in & 1.69 & 0.18 & 2.13 & 0.38 & 1.91 & 0.36 \\
\hline & & Total & 1.53 & 0.22 & 1.93 & 0.36 & $1.73^{b}$ & 0.35 \\
\hline & & $0.017 \times 0.025$-in & 1.61 & 0.28 & 1.93 & 0.28 & $1.77^{a}$ & 0.32 \\
\hline & Total & $0.019 \times 0.025$-in & 1.93 & 0.30 & 2.14 & 0.32 & $2.04^{\beta}$ & 0.32 \\
\hline & & Total & $1.77^{b}$ & 0.33 & $2.04^{a}$ & 0.31 & 1.90 & 0.34 \\
\hline
\end{tabular}

For each activation, means followed by distinct letters differ significantly by means of the analysis of variance (lower case and uppercase are used for independent comparisons), supplemented by Tukey's Multiple Comparison Test, in a level of significance of 5\%. 
Table 8 - Means and standard deviations of load/deflection (L/D) ratio, in $\mathrm{g} / \mathrm{mm}$, generated by teardrop loop, according to the interaction between metal alloy, cross-section and preactivation, during the activation of $1.0 \mathrm{~mm}$ to $5.0 \mathrm{~mm}$.

\begin{tabular}{|c|c|c|c|c|c|c|c|c|}
\hline \multirow{3}{*}{$\begin{array}{c}\text { Activation } \\
(\mathrm{mm})\end{array}$} & \multirow{3}{*}{ Metal alloy } & \multirow{3}{*}{ Cross-section } & \multicolumn{4}{|c|}{ Preactivation } & \multicolumn{2}{|c|}{ Total } \\
\hline & & & \multicolumn{2}{|c|}{$0^{\circ}$} & \multicolumn{2}{|c|}{$40^{\circ}$} & \multirow[b]{2}{*}{ Mean } & \multirow[b]{2}{*}{ SD } \\
\hline & & & Mean & SD & Mean & SD & & \\
\hline \multirow{9}{*}{1.0} & \multirow{3}{*}{ Stainless steel } & $0.017 \times 0.025$-in & 236.00 & 33.62 & 306.00 & 26.08 & $271.00^{b}$ & 46.54 \\
\hline & & $0.019 \times 0.025$-in & 302.00 & 40.25 & 422.00 & 38.99 & $362.00^{\mathrm{a}}$ & 73.45 \\
\hline & & Total & $269.00^{\mathrm{B}}$ & 49.32 & $364.00^{A}$ & 68.67 & 316.50 & 75.90 \\
\hline & \multirow{3}{*}{ Beta-titanium } & $0.017 \times 0.025$-in & 94.00 & 15.17 & 143.00 & 8.37 & $118.50^{d}$ & 28.29 \\
\hline & & $0.019 \times 0.025$-in & 156.00 & 30.50 & 184.00 & 8.94 & $170.00^{c}$ & 25.82 \\
\hline & & Total & $125.00^{\mathrm{D}}$ & 39.79 & $163.50^{c}$ & 23.10 & 144.25 & 37.32 \\
\hline & \multirow{3}{*}{ Total } & $0.017 \times 0.025$-in & 165.00 & 78.78 & 224.50 & 87.83 & 194.75 & 86.75 \\
\hline & & $0.019 \times 0.025$-in & 229.00 & 83.99 & 303.00 & 128.24 & 266.00 & 112.13 \\
\hline & & Total & 197.00 & 85.78 & 263.75 & 114.30 & 230.38 & 105.32 \\
\hline \multirow{9}{*}{2.0} & \multirow{3}{*}{ Stainless steel } & $0.017 \times 0.025$-in & $226.00^{D}$ & 23.82 & $290.50^{C}$ & 11.24 & 258.25 & 38.26 \\
\hline & & $0.019 \times 0.025$-in & $298.00^{\mathrm{B}}$ & 22.80 & $416.00^{A}$ & 18.51 & 357.00 & 65.20 \\
\hline & & Total & 262.00 & 43.86 & 353.25 & 67.70 & 307.63 & 72.62 \\
\hline & \multirow{3}{*}{ Beta-titanium } & $0.017 \times 0.025$-in & $99.00^{\mathrm{F}}$ & 9.78 & $131.00^{\mathrm{EF}}$ & 10.84 & 115.00 & 19.47 \\
\hline & & $0.019 \times 0.025$-in & $156.50^{\mathrm{EF}}$ & 13.87 & $179.00^{\mathrm{E}}$ & 9.62 & 167.75 & 16.35 \\
\hline & & Total & 127.75 & 32.35 & 155.00 & 27.08 & 141.38 & 32.23 \\
\hline & \multirow{3}{*}{ Total } & $0.017 \times 0.025$-in & 162.50 & 69.10 & 210.75 & 84.71 & 186.63 & 79.20 \\
\hline & & $0.019 \times 0.025$-in & 227.25 & 76.67 & 297.50 & 125.68 & 262.38 & 107.54 \\
\hline & & Total & 194.88 & 78.42 & 254.13 & 113.41 & 224.50 & 100.81 \\
\hline \multirow{9}{*}{3.0} & \multirow{3}{*}{ Stainless steel } & $0.017 \times 0.025$-in & $207.33^{c}$ & 16.36 & $274.00^{\mathrm{B}}$ & 6.41 & 240.67 & 37.04 \\
\hline & & $0.019 \times 0.025$-in & $279.00^{\mathrm{B}}$ & 11.28 & $392.00^{\mathrm{A}}$ & 6.06 & 335.50 & 60.16 \\
\hline & & Total & 243.17 & 40.03 & 333.00 & 62.47 & 288.08 & 68.78 \\
\hline & \multirow{3}{*}{ Beta-titanium } & $0.017 \times 0.025$-in & $99.33^{G}$ & 7.51 & $130.00^{\mathrm{F}}$ & 8.16 & 114.67 & 17.77 \\
\hline & & $0.019 \times 0.025$-in & 151.33E & 10.43 & $179.00^{\mathrm{D}}$ & 9.83 & 165.17 & 17.44 \\
\hline & & Total & 125.33 & 28.72 & 154.50 & 27.19 & 139.92 & 31.06 \\
\hline & \multirow{3}{*}{ Total } & $0.017 \times 0.025$-in & 153.33 & 58.17 & 202.00 & 76.21 & 177.67 & 70.55 \\
\hline & & $0.019 \times 0.025$-in & 215.17 & 68.06 & 285.50 & 112.52 & 250.33 & 97.44 \\
\hline & & Total & 184.25 & 69.31 & 243.75 & 102.88 & 214.00 & 91.67 \\
\hline & & $0.017 \times 0.025$-in & $190.25^{c}$ & 11.81 & $254.00^{\mathrm{B}}$ & 3.79 & 222.13 & 34.60 \\
\hline & Stainless steel & $0.019 \times 0.025$-in & $259.25^{\mathrm{B}}$ & 7.27 & $358.00^{\mathrm{A}}$ & 4.11 & 308.63 & 52.34 \\
\hline & & Total & 224.75 & 37.52 & 306.00 & 54.94 & 265.38 & 61.92 \\
\hline & & $0.017 \times 0.025$-in & $96.75^{F}$ & 5.56 & $127.75^{\mathrm{E}}$ & 6.75 & 112.25 & 17.35 \\
\hline 4.0 & Beta-titanium & $0.019 \times 0.025$-in & $144.25^{\circ}$ & 7.53 & $176.00^{c}$ & 9.45 & 160.13 & 18.57 \\
\hline & & Total & 120.50 & 25.80 & 151.88 & 26.58 & 136.19 & 30.15 \\
\hline & & $0.017 \times 0.025$-in & 143.50 & 50.04 & 190.88 & 66.74 & 167.19 & 62.34 \\
\hline & Total & $0.019 \times 0.025$-in & 201.75 & 61.01 & 267.00 & 96.17 & 234.38 & 85.23 \\
\hline & & Total & 172.63 & 61.99 & 228.94 & 89.53 & 200.78 & 81.18 \\
\hline & & $0.017 \times 0.025$-in & $178.20^{c}$ & 10.92 & $235.20^{\mathrm{B}}$ & 3.35 & 206.70 & 30.99 \\
\hline & Stainless steel & $0.019 \times 0.025$-in & $243.00^{\mathrm{B}}$ & 7.28 & $326.60^{A}$ & 3.13 & 284.80 & 44.38 \\
\hline & & Total & 210.60 & 35.26 & 280.90 & 48.27 & 245.75 & 54.71 \\
\hline & & $0.017 \times 0.025$-in & $93.60^{\mathrm{E}}$ & 4.72 & $124.40^{\mathrm{D}}$ & 6.27 & 109.00 & 17.06 \\
\hline 5.0 & Beta-titanium & $0.019 \times 0.025$-in & $137.00^{\mathrm{D}}$ & 5.70 & $169.60^{C}$ & 8.29 & 153.30 & 18.45 \\
\hline & & Total & 115.30 & 23.40 & 147.00 & 24.81 & 131.15 & 28.56 \\
\hline & & $0.017 \times 0.025$-in & 135.90 & 45.29 & 179.80 & 58.59 & 157.85 & 55.72 \\
\hline & Total & $0.019 \times 0.025$-in & 190.00 & 56.21 & 248.10 & 82.96 & 219.05 & 75.13 \\
\hline & & Total & 162.95 & 56.90 & 213.95 & 78.19 & 188.45 & 72.27 \\
\hline
\end{tabular}

For each activation, means followed by distinct letters differ significantly by means of the analysis of variance (lower case and uppercase are used for independent comparisons), supplemented by Tukey's Multiple Comparison Test, in a level of significance of 5\%. 
Table 9 - Means and standard deviations of load/deflection (L/D) ratio, in $\mathrm{g} / \mathrm{mm}$, generated by teardrop loop with helix according to the interaction between metal alloy, cross-section and preactivation, during their activation of $1.0 \mathrm{~mm}$ to $5.0 \mathrm{~mm}$

\begin{tabular}{|c|c|c|c|c|c|c|c|c|}
\hline \multirow{3}{*}{$\begin{array}{c}\text { Activation } \\
\text { (mm) }\end{array}$} & \multirow{3}{*}{$\begin{array}{l}\text { Metal } \\
\text { alloy }\end{array}$} & \multirow{3}{*}{ Cross-section } & \multicolumn{4}{|c|}{ Preactivation } & \multicolumn{2}{|c|}{ Total } \\
\hline & & & \multicolumn{2}{|c|}{$0^{\circ}$} & \multicolumn{2}{|c|}{$40^{\circ}$} & \multirow[b]{2}{*}{ Mean } & \multirow[b]{2}{*}{ SD } \\
\hline & & & Mean & SD & Mean & SD & & \\
\hline \multirow{9}{*}{1.0} & \multirow{3}{*}{ Stainless steel } & $0.017 \times 0.025$-in & 155.00 & 13.23 & 203.00 & 26.83 & $179.00^{b}$ & 32.21 \\
\hline & & $0.019 \times 0.025$-in & 212.00 & 16.81 & 272.00 & 17.89 & $242.00^{a}$ & 35.61 \\
\hline & & Total & $183.50^{\mathrm{B}}$ & 33.25 & $237.50^{\mathrm{A}}$ & 42.25 & 210.50 & 46.22 \\
\hline & \multirow{3}{*}{ Beta-titanium } & $0.017 \times 0.025$-in & 69.00 & 4.18 & 94.00 & 8.22 & $81.50^{d}$ & 14.54 \\
\hline & & $0.019 \times 0.025$-in & 87.00 & 8.37 & 117.00 & 4.47 & $102.00^{c}$ & 17.03 \\
\hline & & Total & $78.00^{\circ}$ & 11.35 & $105.50^{c}$ & 13.63 & 91.75 & 18.66 \\
\hline & \multirow{3}{*}{ Total } & $0.017 \times 0.025$-in & 112.00 & 46.26 & 148.50 & 60.42 & 130.25 & 55.62 \\
\hline & & $0.019 \times 0.025$-in & 149.50 & 67.06 & 194.50 & 82.61 & 172.00 & 76.78 \\
\hline & & Total & 130.75 & 59.28 & 171.50 & 74.29 & 151.13 & 69.47 \\
\hline \multirow{9}{*}{2.0} & \multirow{3}{*}{ Stainless steel } & $0.017 \times 0.025$-in & 163.00 & 8.18 & 198.00 & 18.23 & $180.50^{\mathrm{b}}$ & 22.75 \\
\hline & & $0.019 \times 0.025$-in & 223.00 & 12.55 & 267.50 & 17.14 & $245.25^{a}$ & 27.40 \\
\hline & & Total & $193.00^{\mathrm{B}}$ & 33.16 & $232.75^{\mathrm{A}}$ & 40.25 & 212.88 & 41.28 \\
\hline & \multirow{3}{*}{ Beta-titanium } & $0.017 \times 0.025$-in & 75.00 & 3.06 & 94.00 & 6.75 & $84.50^{d}$ & 11.17 \\
\hline & & $0.019 \times 0.025$-in & 89.00 & 9.45 & 112.00 & 5.97 & $100.50^{c}$ & 14.23 \\
\hline & & Total & $82.00^{\circ}$ & 9.92 & $103.00^{c}$ & 11.23 & 92.50 & 14.91 \\
\hline & \multirow{3}{*}{ Total } & $0.017 \times 0.025$-in & 119.00 & 46.74 & 146.00 & 56.32 & 132.50 & 52.25 \\
\hline & & $0.019 \times 0.025$-in & 156.00 & 71.40 & 189.75 & 82.84 & 172.88 & 77.24 \\
\hline & & Total & 137.50 & 61.72 & 167.88 & 72.51 & 152.69 & 68.22 \\
\hline \multirow{9}{*}{3.0} & \multirow{3}{*}{ Stainless steel } & $0.017 \times 0.025$-in & 167.33 & 7.32 & 198.33 & 14.34 & $182.83^{b}$ & 19.55 \\
\hline & & $0.019 \times 0.025$-in & 220.00 & 13.12 & 267.33 & 14.79 & $243.67^{a}$ & 28.22 \\
\hline & & Total & $193.67^{\mathrm{B}}$ & 29.51 & $232.83^{A}$ & 38.87 & 213.25 & 39.14 \\
\hline & \multirow{3}{*}{ Beta-titanium } & $0.017 \times 0.025$-in & 78.00 & 5.19 & 94.00 & 4.80 & $86.00^{d}$ & 9.66 \\
\hline & & $0.019 \times 0.025$-in & 92.33 & 8.47 & 108.67 & 7.01 & $100.50^{c}$ & 11.31 \\
\hline & & Total & $85.17^{D}$ & 10.04 & $101.33^{c}$ & 9.58 & 93.25 & 12.65 \\
\hline & \multirow{3}{*}{ Total } & $0.017 \times 0.025$-in & 122.67 & 47.46 & 146.17 & 55.90 & 134.42 & 51.89 \\
\hline & & $0.019 \times 0.025$-in & 156.17 & 68.09 & 188.00 & 84.33 & 172.08 & 76.36 \\
\hline & & Total & 139.42 & 59.65 & 167.08 & 72.87 & 153.25 & 67.21 \\
\hline & & $0.017 \times 0.025$-in & 165.00 & 6.31 & 195.00 & 11.59 & $180.00^{b}$ & 18.10 \\
\hline & Stainless steel & $0.019 \times 0.025$-in & 214.25 & 11.85 & 263.25 & 13.62 & $238.75^{a}$ & 28.49 \\
\hline & & Total & $189.63^{B}$ & 27.46 & $229.13^{A}$ & 37.90 & 209.38 & 38.05 \\
\hline & & $0.017 \times 0.025$-in & 82.50 & 5.00 & 94.50 & 4.38 & $88.50^{d}$ & 7.72 \\
\hline 4.0 & Beta-titanium & $0.019 \times 0.025$-in & 96.50 & 8.86 & 109.75 & 6.87 & $103.13^{c}$ & 10.23 \\
\hline & & Total & $89.50^{D}$ & 10.02 & $102.13^{c}$ & 9.70 & 95.81 & 11.58 \\
\hline & & $0.017 \times 0.025$-in & 123.75 & 43.81 & 144.75 & 53.61 & 134.25 & 48.85 \\
\hline & Total & $0.019 \times 0.025$-in & 155.38 & 62.84 & 186.50 & 81.54 & 170.94 & 72.63 \\
\hline & & Total & 139.56 & 55.16 & 165.63 & 70.49 & 152.59 & 63.86 \\
\hline & & $0.017 \times 0.025$-in & 163.20 & 6.61 & 190.80 & 9.01 & $177.00^{b}$ & 16.34 \\
\hline & Stainless steel & $0.019 \times 0.025$-in & 208.80 & 12.15 & 255.20 & 13.61 & $232.00^{\mathrm{a}}$ & 27.31 \\
\hline & & Total & $186.00^{\mathrm{B}}$ & 25.74 & $223.00^{A}$ & 35.64 & 204.50 & 35.72 \\
\hline & & $0.017 \times 0.025$-in & 84.80 & 6.30 & 95.80 & 4.49 & $90.30^{d}$ & 7.76 \\
\hline 5.0 & Beta-titanium & $0.019 \times 0.025$-in & 99.00 & 8.00 & 111.60 & 6.99 & $105.30^{c}$ & 9.71 \\
\hline & & Total & $91.90^{\circ}$ & 10.10 & $103.70^{c}$ & 10.00 & 97.80 & 11.51 \\
\hline & & $0.017 \times 0.025$-in & 124.00 & 41.77 & 143.30 & 50.52 & 133.65 & 46.19 \\
\hline & Total & $0.019 \times 0.025$-in & 153.90 & 58.68 & 183.40 & 76.37 & 168.65 & 67.99 \\
\hline & & Total & 138.95 & 51.89 & 163.35 & 66.29 & 151.15 & 60.04 \\
\hline
\end{tabular}

For each activation, means followed by distinct letters differ significantly by means of the analysis of variance (lower case and uppercase are used for independent comparisons), supplemented by Tukey's Multiple Comparison Test, in a level of significance of 5\%. 


\section{DISCUSSION}

Controlling a force system applied on a tooth is one of the major challenges in biomechanical orthodontic field. It happens because obtaining optimum magnitude of force in combination with the application of an appropriate $\mathrm{M} / \mathrm{F}$ ratio is not an easy task to be achieved. $7,10,13$

Then, a series of studies ${ }^{2,6,11,12,16,25}$ has been performed in order to develop an optimized configuration of orthodontic loops to space closure with a generation of a predictable force system, safe, and consistent with biological mechanisms related to induced tooth movement. The force system produced by these loops depends on a wide range of variables, including design and dimensions of loop, metal alloy used, thickness of wire, conformation of the loop when it is preactivated, its positioning between brackets, and its extent of activation. The control of this forces system is essential to the optimization of inducing tooth movement where the magnitude of the force applied and its direction, the $\mathrm{M} / \mathrm{F}$ ratio, and the constancy of all these factors are important variables which can be determined by the orthodontist during the treatment.

In this study the mechanical test was performed to all the groups of loops with a maxim activation of $5 \mathrm{~mm}$, since it was an experimental work. However, it is important to highlight that despite in clinical setting the stainless steel loops are not activated in such magnitude, the experiments were performed with the intention of evaluating mechanical behaviors which will occur before an imminent permanent deformation. The loops made of beta-titanium wire allow higher amplitude of activation due to mechanical properties inherent of this metal alloy.

In order to stipulate values to the optimum force for anterior tooth retraction, Shimizu ${ }^{20}$ mentioned that the ideal horizontal force for mandibular canine movement is around $120 \mathrm{~g}$, and $150 \mathrm{~g}$ for maxillary canine; for mandibular incisors would be around $240 \mathrm{~g}$ and for maxillary incisors approximately $300 \mathrm{~g}$; finally, $480 \mathrm{~g}$ for mandibular en-masse retraction (incisors and canine), and $600 \mathrm{~g}$ for maxillary en-masse retraction. In this way, it can be seen that light forces are enough for anterior tooth movement, minimizing the biological costs of the mechanotherapy (Table 3).

To compare the values described above with the values of horizontal force described in this work, it must be considered that, for sequential or en-masse retraction, the values obtained through this mechanical test must be multiplied by two, since during the retraction of anterior segment, it is common to use loops bilaterally.

Comparing data presented in Table 4 with optimum values of horizontal force recommended for tooth movement (Table 3), it was found that only teardrop loops in $0.017 \times 0.025$-in beta-titanium wires with no preactivation presented a horizontal force magnitude consistent (94 g) for mandibular canines retraction, that occurs with $1 \mathrm{~mm}$ activation. Favorable magnitudes of force for maxillary canine movement were obtained with activation of $1 \mathrm{~mm}$ of $0.017 \times 0.025$-in beta-titanium loops with $40^{\circ}$ preactivation, and on $0.019 \times 0.025$-in with no preactivation (143.0 g and $156.0 \mathrm{~g}$, respectively). All the stainless steel loops in any amplitude of activation presented excessive magnitudes of force for maxillary and mandibular canines retraction. In incisors movement, only the $0.017 \times 0.025$-in beta-titanium loops with $40^{\circ}$ of preactivation, and the $0.019 \times 0.025$-in with no preactivation, produced appropriate levels of horizontal force for maxillary incisors retraction (286 g and $312 \mathrm{~g}$, respectively), when $1 \mathrm{~mm}$ of activation was present.

For mandibular en-masse retraction, only the $0.017 \times 0.025$-in stainless steel loops with no preactivation bend and with $1 \mathrm{~mm}$ of activation generated reasonable horizontal force magnitude $(472 \mathrm{~g})$. For maxillary en-masse retraction, acceptable magnitudes of force were obtained through the activation of $1 \mathrm{~mm}$ on the $0.017 \times 0.025$-in stainless steel loops with $40^{\circ}$ of preactivation and $0.019 \times 0.025$-in with no preactivation and also by $0.017 \times 0.025$-in beta-titanium loops with no preactivation and with a $3-\mathrm{mm}$ activation (612 g, $604 \mathrm{~g}$ and $596 \mathrm{~g}$, respectively).

Comparing the data presented on Table 5 with optimum force values recommended for tooth movement (Table 3), it was found that the teardrop loops with helix in $0.017 \times 0.025$-in and $0.019 \times 0.025$-in beta-titanium with $40^{\circ}$ of bend preactivation and activated at $1 \mathrm{~mm}$ presented magnitudes of horizontal force (94 g and $117 \mathrm{~g}$, respectively) coherent for mandibular canine retraction. For maxillary canine retraction, the $0.017 \times 0.025$-in stainless steel loops with no preactivation bends and activated by $1 \mathrm{~mm}$ generated acceptable levels of horizontal force, as well as beta- 
titanium with an activation of $2 \mathrm{~mm}(155.0 \mathrm{~g}$ and $150.0 \mathrm{~g}$, respectively). Magnitudes of force appropriate for mandibular incisors movement were obtained with an activation of $1 \mathrm{~mm}$ on the $0.019 \times 0.025$-in beta-titanium loops with helix and $40^{\circ}$ of preactivation bend $(234.0 \mathrm{~g})$. In maxillary incisors movement, both 0.017 $\mathrm{x}$ 0.025-in stainless steel loop with no preactivation bend, and activated by $1 \mathrm{~mm}$, as 0.017 x 0.025-in betatitanium with no preactivation bend and activated by $2 \mathrm{~mm}$ generated magnitudes of horizontal force clinically acceptable (310 g and $300 \mathrm{~g}$, respectively). For incisors and canines retraction, $0.017 \times 0.025$-in beta-titanium loops with helix and activated by $3.0 \mathrm{~mm}$ presented favorable levels of force for the movement in mandibular arch when they were not preactivated (468 g). The same favorable outcome for the movement of these teeth in the maxilla happened when the archwire presented $40^{\circ}$ of preactivation $(564 \mathrm{~g})$.

In this way, it was observed that teardrop loops and teardrop loops with helix, when they were made in stainless steel, did not provide favorable horizontal

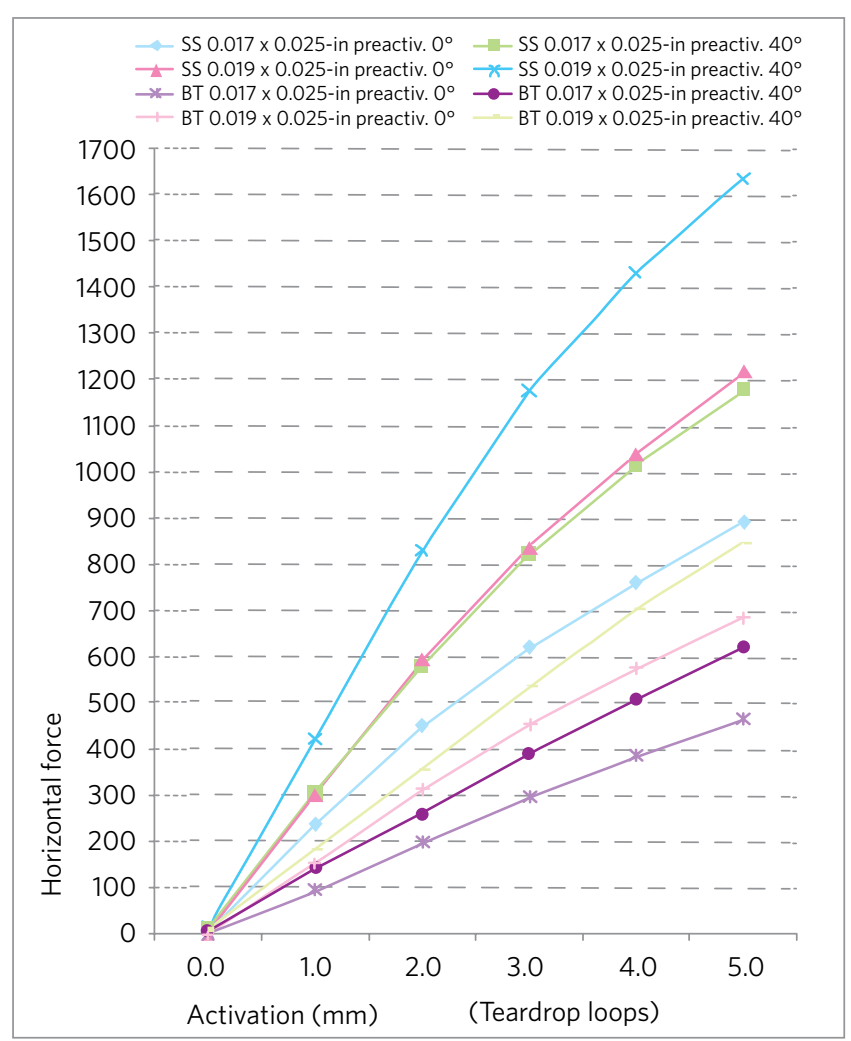

Figure 6 - Average of the magnitudes of horizontal force $(\mathrm{g})$ generated by teardrop loops made according to interactions between metal alloy (stainless steel and beta-titanium), cross-section (0.017 $\times 0.025$-in and $0.019 \times 0.025$-in), and preactivation $\left(0^{\circ}\right.$ and $\left.40^{\circ}\right)$. force level for anterior tooth movement when they were activated more than $1 \mathrm{~mm}$. Loops with same design made in beta-titanium allows $3 \mathrm{~mm}$ clinical activation, maintaining the acceptable levels of force for anterior teeth.

In general way, teardrop loops with helix generated lower magnitudes of horizontal force when they were compared with teardrop loops. It occurs due to the higher amount of wire incorporated in the loop, providing the possibility to apply lower levels of horizontal force with higher limits of elasticity during the loop activation (Figs 6 and 7). It confirms the findings of Chaconas et al, ${ }^{4}$ and Schillai and Lehmann. ${ }^{19}$

Among the variables analyzed in this study, metal alloy composition was the one that presented the highest influence on horizontal force magnitude. The beta-titanium archwire a reduction of 50\% in the magnitude of horizontal force delivered by teardrop loops, and of $54,5 \%$ in teardrop loops with helix.

Cross-sectional dimension also produced a marked influence in the magnitude of horizontal

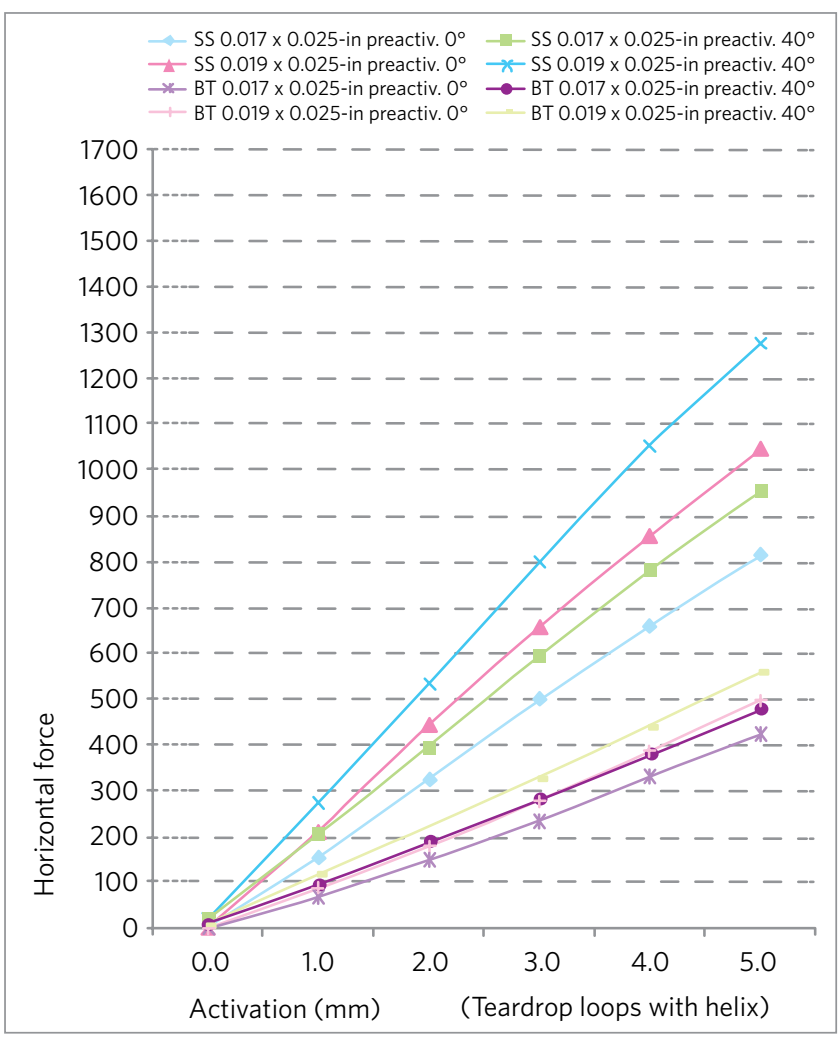

Figure 7 - Average of the magnitudes of horizontal force $(\mathrm{g})$ generated by teardrop loops with helix made according to interactions between metal alloy (stainless steel and beta-titanium), cross-section $(0.017 \times 0.025$-in and $0.019 \times 0.025-\mathrm{in})$, and preactivation $\left(0^{\circ}\right.$ and $\left.40^{\circ}\right)$ 
force delivered by the loops. In those made in stainless steel, the $0.017 \times 0.025-$ in dimension generated force levels around 26\% lower than the $0.019 \times 0.025$-in. In the beta-titanium loops, this reduction was around $22.5 \%$.

Regarding the preactivation, it has shown a small influence in the magnitude of force delivered by the loops. However, it presented statistical significance. Preactivation bends, in general way, provide higher amount of horizontal force, and this finding was also reported by Chen et al, ${ }^{5}$ Raboud et $\mathrm{al}^{16}$ and Shimizu et al. ${ }^{21}$ Still, the increase on the magnitude of horizontal force was not as intense as observed by these authors; it was performed an appropriate distribution of preactivation bends, going from occlusal to gingival aspect, according to the recommended by Braun and Garcia, ${ }^{1}$ and Halazonetis. ${ }^{7}$

These high levels of horizontal force delivered by preactivated loops can be explained because, many times, the neutral state of activation is very difficult to be achieved. ${ }^{3}$ It occurs since loops, after engaged on the anchorage teeth brackets, can present certain inherent deformation due to the preactivation bends performed (e.g. the overlapping of their vertical rods and consequently decreasing the inter-bracket distance) and provide the appearance of residual force prior to their activation.

Another relevant aspect to be observed is the $\mathrm{M} / \mathrm{F}$ ratio generated by the teardrop loops. The $\mathrm{M} / \mathrm{F}$ ratio is the relation between the amount of moment and the force applied on a tooth, considering the center of resistance and determining the rotation center. It is one of the most important characteristics of space closure device because it determines the center of rotation and therefore, the direction that the tooth will be moving.

Investigating the relation between $\mathrm{M} / \mathrm{F}$ ratio and tooth rotation centers, Tanne et $\mathrm{al}^{23}$ could observe that the necessary $\mathrm{M} / \mathrm{F}$ ratio to correct the angulation of a single-rooted tooth was 9.53, and the center of rotation was the incisal edge. For translation movement, the $\mathrm{M} / \mathrm{F}$ ratio required was 8.93 and 6.52 for controlled tipping (with center of rotation in the apex).Values lower than 6.52 would result only on uncontrolled tipping movement.

In observation of results presented in Tables 6 and 7, it was found that all teardrop designs evaluated generate a $\mathrm{M} / \mathrm{F}$ ratio relatively low when they were activated, which provides only uncontrolled tipping. The same happened with teardrop loops, all the configurations of teardrop loops with helix did not produced an increased $\mathrm{M} / \mathrm{F}$ ratio. However, teardrop loops with stainless steel helixes and teardrop loops with $0.019 \times 0.025$-in beta-titanium helixes and $40^{\circ}$ preactivation bend, resulted on medium values of $\mathrm{M} / \mathrm{F}$ ratio when activated by $1 \mathrm{~mm}$. This medium values are enough to perform a controlled tipping movement.

Based on the results, it is possible to verify that when preactivation bends are present the loops tested in this study generate low $\mathrm{M} / \mathrm{F}$ ratio, insufficient to provide root correction and translational movement, important in the space closure mechanics. In the absence of preactivation bends, the $\mathrm{M} / \mathrm{F}$ ratio generated was even lower and all loops provide only the uncontrolled tipping demonstrated in Figures 8 and 9.

Among the variables analyzed in this study, the preactivation bends presented higher impact in the $\mathrm{M} / \mathrm{F}$ ratio exerted by the teardrop loops. Loops with $40^{\circ}$ of preactivation resulted on significantly higher $\mathrm{M} / \mathrm{F}$ ratio than loops without preactivation.

Another variable that presented some influence on $\mathrm{M} / \mathrm{F}$ ratio was the metal alloy composition. It was observed that, in general, loops made of stainless steel wire resulted in higher $\mathrm{M} / \mathrm{F}$ values.

With regard to cross-sectional dimension, it presented a very low influence on the M/F ratio generated by orthodontic loops for space closure. For teardrop loops with helix, the specimens made of $0.019 \times 0.025$-in archwire in general showed higher levels of $\mathrm{M} / \mathrm{F}$ values. On the other hand, higher $\mathrm{M} / \mathrm{F}$ values was generally seen in the specimens with $0.017 \times 0.025$-in loops in the group with no helix.

Generally, the cross-sectional dimension of a wire and its modulus of elasticity present few effects in the $\mathrm{M} / \mathrm{F}$ ratio generated by loops with no preactivation. However, the use of metal alloys with lower modulus of elasticity, for example beta-titanium, allows an incorporation of preactivation bends of higher intensity. So, this alloy is elected when it is aimed an increase on $\mathrm{M} / \mathrm{F}$ ratio.

In this study, teardrop loops and teardrop loops with helix in beta-titanium wires presented values of $\mathrm{M} / \mathrm{F}$ lower than teardrop loops in stainless steel. It happens due to the incorporation of an equal amount of preactivation. 


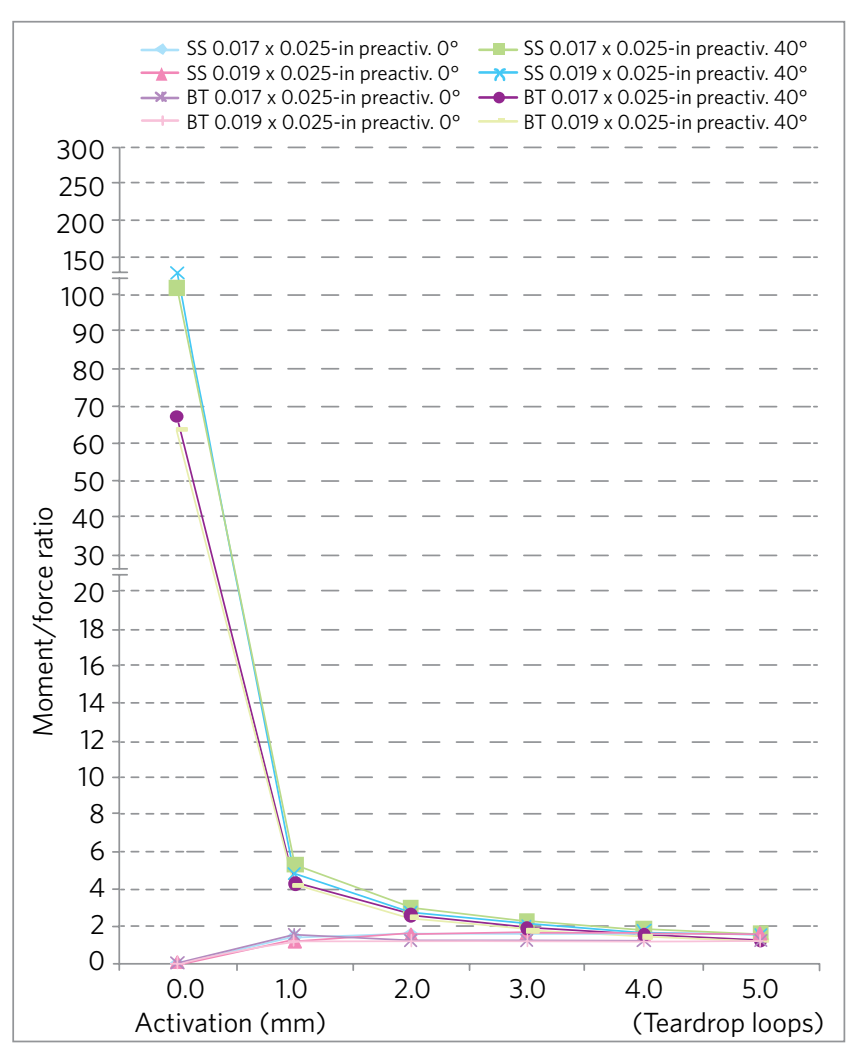

Figure 8 - Average of magnitudes in moment/force ratio $(\mathrm{mm})$ generated by teardrop loops made according to interactions between metal alloy (stainless steel and beta-titanium), cross-section (0.017 × 0.025-in and $0.019 \times 0.025$-in), and preactivation $\left(0^{\circ}\right.$ and $\left.40^{\circ}\right)$.

However, beta-titanium loops could present higher values of $\mathrm{M} / \mathrm{F}$, since this alloy permits preactivation bends 100\% higher than those inserted in stainless steel loops, with no permanent deformation when they are active. ${ }^{13}$ Therefore, when higher $\mathrm{M} / \mathrm{F}$ ratio is needed, the intensity of preactivation bends could be increased, always looking after to avoid a drastic elevation of horizontal force values.

This same intensity of preactivation of betatitanium loops was performed in this study only for comparative purposes, since there was no data in orthodontic literature related to the intensity of preactivation recommended for these design of beta-titanium loops. Also, there was no data about the force system generated by these designs of loop in any preactivation degree. For stainless steel, the literature recommends a $20^{\circ}$ preactivation bend on each side of the loop, in a total of $40^{\circ}$ to $45^{\circ}$, in order to achieve an appropriate $\mathrm{M} / \mathrm{F}$ ratio. ${ }^{4,15,16,21}$

In addition to the highlighted characteristics, the dissipation rate of horizontal force provided by the loop along its deactivation, which is called $\mathrm{L} / \mathrm{D}$ ratio,

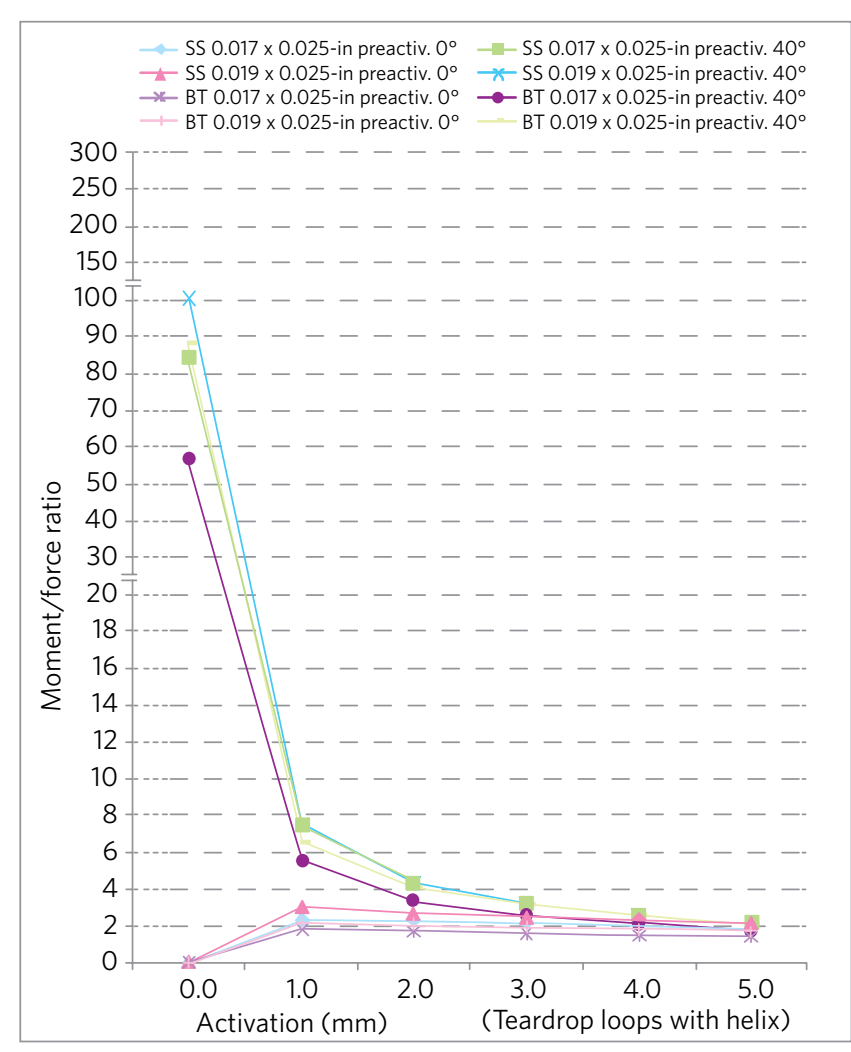

Figure 9 - Average of magnitudes in moment/force ratio $(\mathrm{mm})$ generated by teardrop loops with helix made according to interactions between metal alloy (stainless steel and beta-titanium), cross-section (0.017 $\times 0.025$-in and $0.019 \times 0.025$-in), and preactivation $\left(0^{\circ}\right.$ and $\left.40^{\circ}\right)$.

constitutes an aspect of great interest to the evaluation of mechanical properties of space closure loops. A low $\mathrm{L} / \mathrm{D}$ value is important in a loop since it allows the orthodontist to apply light, continuous and controlled forces. Loops with high $\mathrm{L} / \mathrm{D}$ value generate excessive horizontal force and dissipates quickly its force, becoming difficult to establish magnitudes of ideal forces.

It was possible to observe by the results shown in Tables 8 and 9 that, in general way, the L/D ratio generated by teardrop loops and teardrop loops with helixes suffered higher influence of metal alloy composition, and stainless steel presented higher $\mathrm{L} / \mathrm{D}$ ratio when it was compared to beta-titanium. Related to cross-section dimension and preactivation, the $0.019 \times 0.025$-in loops, as well as those with $40^{\circ}$ preactivation, presented higher values of $\mathrm{L} / \mathrm{D}$ ratio. It was also found that lower $\mathrm{L} / \mathrm{D}$ ratios were produced, in general way, by teardrop loops with helixes. The increase in the amount of wire on loops design influence the elastic characteristics of the same loops, decreasing magnitudes of force released and consequently its $\mathrm{L} / \mathrm{D}$ ratio. 


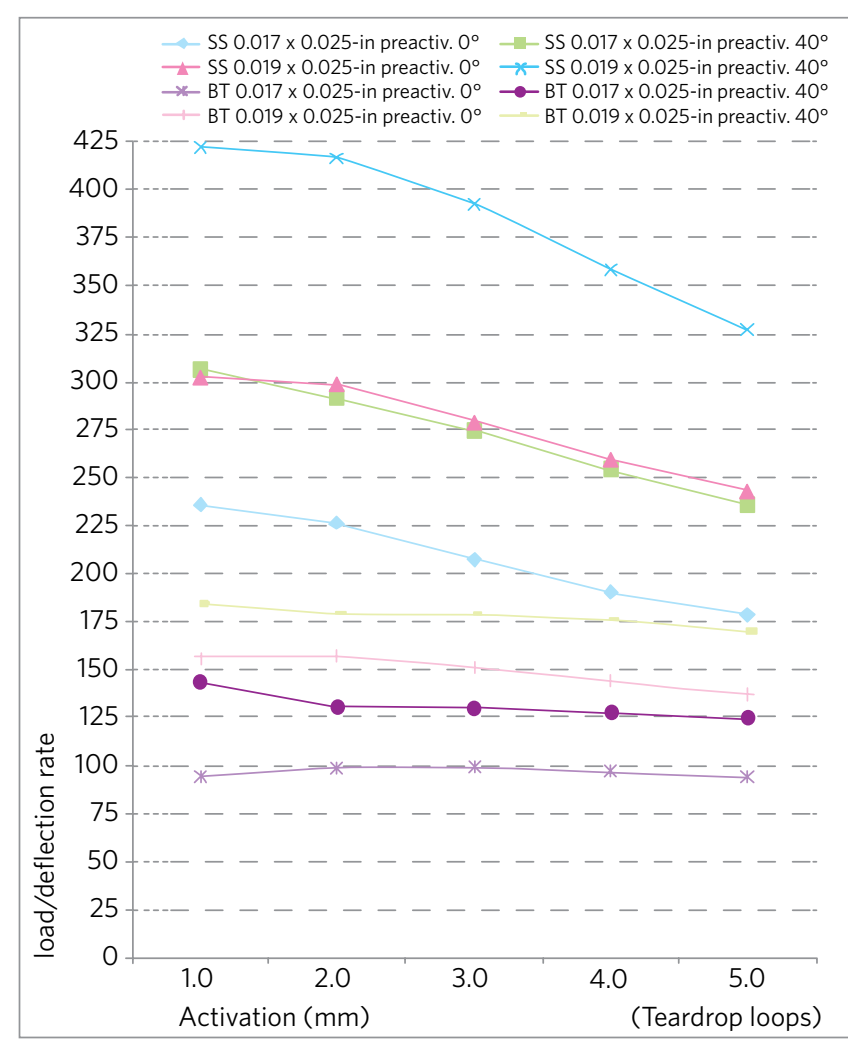

Figure 10 - Average of magnitudes of load/deflection $(\mathrm{g} / \mathrm{mm})$ ratio generated by teardrop loops made according to interactions between metal alloy (stainless steel and beta-titanium), cross-section (0.017 × 0.025-in and $0.019 \times 0.025-$ in), and preactivation $\left(0^{\circ}\right.$ and $\left.40^{\circ}\right)$.

Among the variables analyzed in this study, metal alloy composition presented the highest impact on $\mathrm{L} / \mathrm{D}$ ratio, followed by cross-sectional dimension and, lastly, the preactivation. It was noticed that the $\mathrm{L} / \mathrm{D}$ ratio is a relatively continuous property and has the tendency to softly decrease as the activation of closure loops increases (Figs 10 and 11).

In a separate analysis regarding the presence of helixes in the force system of closing loops hereby tested, it was observed a reduction in the magnitude of horizontal forces around 23\% for stainless steel loops, and of 30\% for beta-titanium loops. Concomitantly with the reduction in the magnitude of horizontal forces by the incorporation of helixes, a reduction occurred in the $\mathrm{L} / \mathrm{D}$ ratio basically on the same level. Related to the $\mathrm{M} / \mathrm{F}$ ratio, the addition of helixes on loops design provided higher magnitudes.

The results in this study show that teardrop loops and teardrop loops with helixes can easily produce force levels high enough to provide tooth movement.

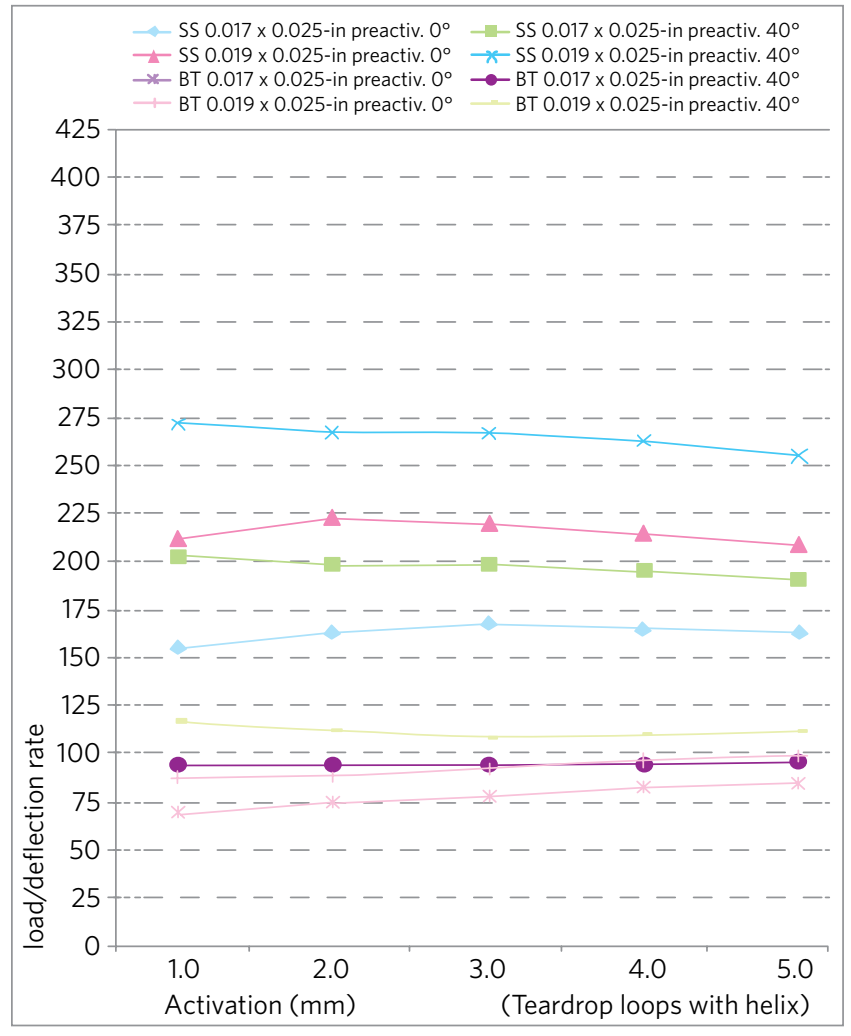

Figure 11 - Average of magnitudes of load/deflection $(\mathrm{g} / \mathrm{mm})$ ratio generated by teardrop loops with helixes made according to interactions between metal alloy (stainless steel and beta-titanium), cross-section (0.017 x 0.025-in and $0.019 \times 0.025$-in), and preactivation $\left(0^{\circ}\right.$ and $\left.40^{\circ}\right)$.

Additionally, due to low $\mathrm{M} / \mathrm{F}$ ratio, the tooth movement achieved is inclination and not translation or root correction. In order to increase the magnitude of moment generated, preactivation bends can be inserted, however, the intensity of the bends performed in this study was insufficient to create a high enough $\mathrm{M} / \mathrm{F}$ ratio.

In this way, it can be observed that the biomechanical performance of orthodontic loops for space closure depends on a series of factors inherent to the configuration of such devices.

\section{CONCLUSIONS}

a) In general, teardrop loops with helixes produced lower magnitudes of horizontal force and $\mathrm{L} / \mathrm{D}$ ratios than teardrop loops. In a preactivation of $40^{\circ}$, the activation of some teardrop loops with helixes generated $\mathrm{M} / \mathrm{F}$ ratio high enough to allow uncontrolled tipping and controlled tipping movements, while the teardrop loops allow only the uncontrolled 
tipping when activated. In the absence of preactivation bends, all loops produced low $\mathrm{M} / \mathrm{F}$ ratios, which allows only uncontrolled tipping when active.

b) Analyzing the effects of cross-sectional dimension, preactivation and metal alloy on the force systems produced, it was possible to conclude that:

» The increase in the cross-sectional dimension of the orthodontic wire provides an increase in magnitude of horizontal force and in $\mathrm{L} / \mathrm{D}$ ratio, providing a small effect on $\mathrm{M} / \mathrm{F}$ ratio generated by the loops.
" The insertion of preactivation bends in loops provides an increase in $\mathrm{M} / \mathrm{F}$ values, horizontal force and $\mathrm{L} / \mathrm{D}$ ratios.

"Related to metal alloy composition, loops made of beta-titanium produced lower magnitudes of horizontal force, $\mathrm{L} / \mathrm{D}$ and $\mathrm{M} / \mathrm{F}$ ratios.

c) Among all the analyzed variables, the one which presented higher influence in the horizontal force and L/D ratio was the metal alloy composition. The $\mathrm{M} / \mathrm{F}$ ratio have shown to be more affected by preactivation of closing loops. 


\section{REFERENCES}

1. Braun S, Garcia JL. The Gable bend revisited. Am J Orthod Dentofacial Orthop. 2002;122(5):523-7

2. Burstone $\mathrm{CJ}$. The segmented arch approach to space closure. Am J Orthod Dentofacial Orthop. 1982;82(5):361-78

3. Burstone $\mathrm{CJ}$, Koenig HA. Optimizing anterior and canine retraction. Am J Orthod. 1976:70(1):1-19.

4. Chaconas SJ, Caputo AA, Hayashi RK. Effects of wire size, loop configuration and gabling on canine-retraction springs. Am J Orthod 1974;65(1):58-66.

5. Chen J, Markham DL, Katona TR. Effects of T-loop geometry on its forces and moments. Angle Orthod 2000;70(1):48-51.

6. Faulkner MG, Fuchshuber P, Haberstock D, Mioduchowski A. A parametric study of the force/moment system produced by T-loop retraction springs. J Biomech. 1989;22(6-7):637-47.

7. Halazonetis DJ. Understanding orthodontic loop preactivation. Am J Orthod Dentofacial Orthop. 1998;113(2):237-41.

8. Jarabak JR, Fizzel JA. Aparatologia del arco de canto com alambres delgados. Buenos Aires: Mundi; 1975. 612 p.

9. Lino AP. Avaliação de propriedades mecânicas de algumas formas da alça de retração ortodôntica, em função do tratamento térmico [tese]. São Paulo: Universidade de São Paulo: 1970.

10. Manhartsberger C, Morton JY, Burstone CJ. Space closure in adults using the segmented arch technique. Angle Orthod. 1989:59(3):205-10.

11. Martins RP, Buschang PH, Gandini LG Jr. Group A T-loop for differential moment mechanics: an implant study. Am J Orthod Dentofacial Orthop. 2009:135(2):182-9.

12. Martins RP, Buschang PH, Viecilli R, dos Santos-Pinto A. Curvature versus $v$-bends in a group B titanium T-loop spring. Angle Orthod. 2008:78(3):517-23.

13. Nanda R, Diaz MAT. Orthodontic space closure. Dent Clin North Am. 1981:25(1):95-107.

14. Scalza Neto P, Mucha JN, Chevitarese O. Mola de fechamento de espaços em Ortodontia em forma de lágrima: desempenho em tração. Rev Bras Odontol. 1985:42(6):22-9

15. Proffit WR. Ortodontia contemporânea. 3 a ed. Rio de Janeiro: Guanabara Koogan; 2002. 677 p
16. Raboud DW, Faulkner MG, Lipsett AW, Haberstock DL. Three-dimensional effects in retraction appliance design. Am J Orthod Dentofacial Orthop. 1997:112(4):378-92

17. Reitan K. Some factors determining the evaluation of forces in orthodontics. Am J Orthod Oral Surg. 1957:43(1):32-45.

18. Ricketts RM. Bioprogressive therapy as an answer to orthodontic needs. Part II. Am J Orthod. 1976;70(4):359-97.

19. Schillai G, Lehmann KM. Untersuchung über die Beziehung zwischen Aktivierungskraft und Auslenkung bei versciedenen Zahnzugfedern Fortschr Kieferorthop. 1989;50(3):172-8

20. Shimizu RH. Fechamento de espaços após exodontias de primeiros prémolares [dissertação]. Araraquara (SP): Universidade Estadual Paulista; 1995

21. Shimizu RH, Sakima T, Santos-Pinto A, Shimizu IA. Comportamento mecânico da mola Bull modificada durante o fechamento de espaços em Ortodontia. Rev Dental Press Ortod Ortop Facial. 2002;7(2):13-24.

22. Smith RJ, Burstone CJ. Mechanics of tooth movement. Am J Orthod. 1984:85(4):294-307.

23. Tanne K, Koening HA, Burstone CJ. Moment to force ratios and the center of rotation. Am J Orthod Dentofacial Orthop. 1988;94(5):426-31.

24. Thiesen $G$, et al. Avaliação das forças liberadas por diferentes tipos de molas de fechamento de espaço utilizadas em Ortodontia. Ortod Gaúch. 2001;5(2):86-91

25. Thiesen G, Rego MVNN, Menezes LM, Shimizu RH. A utilização de diferentes configurações de molas T para a obtenção de sistemas de força otimizados. Rev Dental Press Ortod Ortop Facial. 2006:11(5):57-77.

26. Toti JIS, Sato K. Estudo comparativo das propriedades mecânicas da alça de retração para dentes anteriores (tipo Bull modificada), utilizando fios de Stainless steel de diferentes marcas e espessuras. Ortodontia. 1992:25(2):27-36

27. Tweed CH. Clinical Orthodontics. 3th ed. Saint Louis: Mosby; 1966. v. 2 $946 \mathrm{p}$.

28. Yang TY, Baldwin JJ. Analysis of space closing springs in orthodontics. J Biomech. 1974;7(1):21-8. 